\title{
Cost minimization of large- scale infrastructure for electricity generation and transmission
}

\section{Journal Article}

\section{Author(s):}

Märkle-Huß, Joscha; Feuerriegel, Stefan; Neumann, Dirk

Publication date:

2019-10

Permanent link:

https://doi.org/10.3929/ethz-b-000343583

\section{Rights / license:}

Creative Commons Attribution-NonCommercial-NoDerivatives 4.0 International

Originally published in:

Omega 96, https://doi.org/10.1016/j.omega.2019.05.007 


\title{
Cost minimization of large-scale infrastructure for electricity generation and transmission
}

\author{
Joscha Märkle-Huß ${ }^{\mathrm{a}, *}$, Stefan Feuerriegel ${ }^{\mathrm{b}}$, Dirk Neumann ${ }^{\mathrm{a}}$ \\ ${ }^{a}$ Chair for Information Systems Research, University of Freiburg, Platz der Alten Synagoge, 79098 Freiburg, \\ Germany \\ ${ }^{b}$ ETH Zurich, Weinbergstr. 56/58, 8092 Zurich, Switzerland
}

\begin{abstract}
Electricity infrastructure confronts societies with immense costs as it must ensure the generation of power and its transmission to locations with consumption requirements. We minimize these costs by formulating an electricity generation and transmission problem that facilitates the design of electricity infrastructure on a macro level. Our problem specifies the capacity, type, and location of power plants and, at the same time, determines the appropriate arrangement of high-voltage transmission lines in order to fulfill the demand of individual cities. We specifically incorporate the non-linear nature of cost functions for power generation that are common in practice. This results in a mixed integer non-linear problem, for which the branch-and-reduce solver from GAMS exceeds runtime constraints, even for small instances with 25 locations. As a remedy, we develop heuristics based on the reduced variable neighborhood search and the greedy randomized adaptive search procedure (GRASP). Their performance enables us to address large-scale problems that arise in real-world applications. We demonstrate this with an actual, nationwide example that spans all 4,537 municipalities in Germany.
\end{abstract}

Keywords: Energy, Electricity generation and transmission planning, Neighborhood heuristics, Metaheuristics, Variable neighborhood search, Real-world application

Declarations of interest: none

\section{Introduction}

Electricity infrastructure burdens societies with immense costs. According to expert estimates, the present value of the U. S. electricity system from 2010 to 2040 stands at about $\$ 6.2$ trillion (RMI,

\footnotetext{
${ }^{*}$ Corresponding author. Mail: mjoscha@gmail.com;

Email addresses: mjoscha@gmail.com (Joscha Märkle-Huß), sfeuerriegel@ethz.ch (Stefan Feuerriegel), dirk.neumann@is.uni-freiburg.de (Dirk Neumann)
} 
2011). In Germany, the investment costs of the electricity network in 2015 amounted to $€ 2.4$ billion and a total of $€ 24.2$ billion was paid to providers of renewable energy (Bundesnetzagentur, 2016). Furthermore, multiple studies indicate an increase in generation plant cost in Europe by a factor of 2.6 between 2000 and 2013 and find that 3 out of 4 power plants overrun their originally projected construction costs (Sovacool et al., 2014). Hence, it is inevitable for individuals, businesses, organizations, and societies as whole to seek cost reductions by optimizing the infrastructure of electricity systems (Capros et al., 1988; Samouilidis et al., 1984).

Strategic planning of electricity infrastructure presents a highly complex task, as one must simultaneously decide upon both the location of power plants and the design of the transmission network in order to come up with a cost-efficient solution (Hobbs, 1995). The challenges include, for instance, the fact that the costs of power generation are variable and location-specific. In addition, generation and transmission are highly intertwined, yet subject to competition (Vasin \& Dolmatova, 2016) and long-term planning (Wang \& Deng, 2018). Moreover, strategic decisions must incorporate the different costs for initial investments and operation, as well as the physical constraints of electricity (Antunes \& Gomes, 2009). In realistic settings, this translates into large, complex decision problems involving millions of constraints.

We facilitate the design of large-scale electricity infrastructure by proposing an optimization problem that minimizes the combined costs of power generation and transmission. More specifically, we are given a set of locations, i.e. cities or municipalities, each coming with a certain demand for electricity. The model then determines the optimal capacity, type, and location of power plants, along with corresponding transmission lines, in order to fulfill the electricity demand at each location. In the following, we refer to this as the electricity generation and transmission problem, or EGTP for short. This problem supports practitioners and policy-makers in the complex undertaking of designing cost-efficient electricity systems.

The EGTP adapts to the specific requirements and features of electricity infrastructure. The model thus allows for different types of electricity sources, such as solar or nuclear power plants. Furthermore, it incorporates a non-linear cost function for power generation infrastructure in order to account for the actual costs and physical constraints in practice (Black and Veatch, 2011; Christensen \& Greene, 1976; Walla \& Schneeberger, 2008). That is, as size increases, the unit cost of electricity first decreases due to economies of scale. However, this reduction is limited, as every generation type features a unique, optimal size at which unit costs are lowest. If plants are larger than this optimal size, unit costs increase again due to physical constraints. 
For the above reasons, the EGTP differs in several aspects from the simple plant location problem (SPLP; see Krarup \& Pruzan, 1983), as well as plant location problems with multiple facilities (Ghiani et al., 2002). As mentioned earlier, the EGTP distinguishes between different energy carriers in use for power generation, while the SPLP optimizes over a single type of production. Second, we specifically incorporate varying unit cost for different plant sizes. This results in a non-linear objective function in contrast to the usually linear specifications in the SPLP. Third, transmission costs scale with the net-energy transferred and include an additional fixed component. Furthermore, we are interested in high-voltage transmission infrastructure (i.e. connecting cities) which introduces a set of physical constraint that differs from the usual mesh networks of low-voltage distribution grids (i. e. connecting household within a single city or district). Considerable research, especially in electrical engineering, has been spent on designing distribution infrastructure such as bus systems, whereas the high-voltage transmission setting has received less attention.

The formulation of EGTP presents a problem from mixed-integer non-linear programming (MINLP) and can thus be solved optimally by, for instance, the branch-and-reduce approach (Tawarmalani \& Sahinidis, 2005). However, our computational experiments show that branch-andreduce (even including customized variants for convex MINLP or with linearization) quickly exceeds practical runtime limits, even for small problem instances with $N \geq 25$ locations. This presents a severe barrier to solving large-scale scenarios with $N \geq 1000$ nodes, which arise in practice. In fact, we later show that EGTP is NP-hard. This thus motivates the development of a tailored heuristic exploiting the geometric structure. These heuristics are based on a centralized/decentralized construction, neighborhood search, and the greedy randomized adaptive search procedure (GRASP). These heuristics allow us to solve a real-word case study with actual costs for electricity generation and high-voltage transmission infrastructure that spans all 4,537 municipalities in Germany.

This work entails several practical implications. First, the EGTP presents a holistic approach to electricity infrastructure planning with the combined focus on both power plants and the transmission network. Our problem specifically supports practitioners grappling with the complex design decisions of electricity systems for whole states or nations. The results of our optimization problem may reveal considerable differences between actual and optimal system design. The EGTP thereby provides strategic guidance for practitioners seeking to improve the power infrastructure with the goal of minimizing costs. The problem can easily be extended by additional constraints that reflect regulations or policies, such as a minimum production quota for renewable energy sources. Accordingly, the insights gained from our problem can provide valuable decision support for policy-makers, 
planners of infrastructure expansions, and all remaining stakeholders in electricity systems.

This paper is organized as follows. Section 2 provides an overview of previous research concerning general plant location problems, as well as specific work related to the design of electricity generation

and transmission infrastructure. Section 3 then introduces the specification of our EGTP problem, for which we propose different heuristics in Section 4. The corresponding performance is compared in Section 5, while Section 6 presents results from a large-scale case study with real-world data. Finally, Section 7 concludes.

\section{Related work}

This section reviews previous work related to plant location problems and, in particular, strategic infrastructure planning for large-scale electricity systems.

\subsection{Plant location problems}

The selection of facility locations and delivery routes has a long history within operational research. Especially noteworthy is the simple plant location problem (Krarup \& Pruzan, 1983; Sridharan, 1995), which minimizes the production and transportation costs for a single product by choosing the optimal placement for plants from a finite set of locations. The uncapacitated SPLP describes a problem where the plant size is unlimited. Thus, any plant may produce an arbitrary number of products. In contrast, the capacitated SPLP features a limit on plant size.

A common extension is the capacitated plant location problem with multiple facilities in the same site (e.g. Ghiani et al., 2002; Nickel \& da Gama, 2015; Sabet et al., 2019). Here multiple copies of the same plant type may exist in one location. Such problems have been utilized for determining the optimal locations of, e.g., battery swapping stations for electric vehicles (Sun et al., 2018), emergency response facilities (Adenso-Díaz \& Rodríguez, 1997; Paul et al., 2017) and pharmaceutical companies (Meijboom \& Obel, 2007). Some problems model distribution costs (e.g. Albareda-Sambola et al., 2012; Darvish et al., 2016) or even location routing (Albareda-Sambola et al., 2012; Schiffer \& Walther, 2018), yet these works are tailored to the unique characteristics of logistics and not transmission infrastructure as in our research. Other works specifically address the question of sizing (e.g. Chen, 2013). However, a key difference becomes evident, since our problem must simultaneously identify the optimal location and sizing of multiple plants, while additionally considering the transmission infrastructure (note that, in electricity transmission, flows in opposite direction are not simply added but actually cancel out). 
Similar to the EGTP, plant location problems frequently incorporate economies of scale, since larger plants benefit from efficiency gains (Baumgartner et al., 2012; Christensen \& Greene, 1976). This lowers the total costs per unit, which is commonly modeled by splitting the costs into a fixed and variable component (Feldman et al., 1966; Manne, 1964). The variable part is typically assumed to be linear and thus provides no limits to efficiency. One could potentially consider the latter by exchanging the linear term with a non-linear function; however, this is rarely practically relevant in physical facility problems.

There are various approaches to addressing economics of scale in energy research; however, these are highly problem-specific and thus not directly applicable to our problem. Dornburg \& Faaij (2001) present a bottom-up calculation and demonstrate that the unit cost of electricity generation decreases due to economies of scale in biomass fuel preprocessing and transport, as well as the heat distribution for combined heat and power plants. Similarly, Singh (1990) investigates economies of scale within coal-thermal power plants. The inclusion of economies of scale in a location problem may be solved using non-linear cost curves, as shown in Fleten et al. (2007) for an optimization problem with only one source and load. However, none of the known plant location models actually makes use of this.

The SPLP is formulated as mixed-integer linear programming and has been proven to be NPhard (Krarup \& Pruzan, 1983). Hence, different techniques and heuristics have been developed for solving plant location problems. Among others, Prodhon \& Prins (2014) and Jarboui et al. (2013) propose the use of a variable neighborhood search, since it entails high computational performance. The GRASP metaheuristic can provide a beneficial choice in this case, as it should increase the likelihood of finding a global rather than a local optimum due to its multi-start characteristic and randomization (Duhamel et al., 2010; Resende \& Ribeiro, 2010). This motivates the choice of the optimization methods developed in this paper, as we later adopt the aforementioned techniques used for solving plant location problems and modify them to handle the geometric properties of the EGTP.

Extensive decision problems are located in the broader realm of plant problems, but do not address the unique characteristics of investment decisions for large-scale electricity generation and transmission infrastructure. For instance, there are works that study operational issues such as dispatching (Zugno \& Conejo, 2015) or valuation problems (Ernstsen \& Boomsma, 2018), including the effects of different support schemes (Boomsma et al., 2012). Other research also considers uncertain electricity prices (Zhan et al., 2017) and contributes equilibrium approaches as tailored 
expansion strategies for renewable generation (Pineda et al., 2018). Furthermore, prior literature has leveraged electricity drivers in regular production planning (Golari et al., 2017).

We later develop a specific multi-location planning problem which involves both facilities and a network layout, while considering non-linear costs due to economics of scale and the physical properties of electricity transmission. As the distinguishing characteristic of our problem, the objective function consists of costs that entail a fixed and a (non-linear) variable component.

\subsection{Strategic planning of electricity infrastructure}

The predominant goal in the strategic design of electricity infrastructure is the reduction of investments and/or operational costs (Antunes et al., 2004; Antunes \& Gomes, 2009; Grimm et al., 2016; Guo et al., 2016; Hobbs, 1995; Huppmann \& Egerer, 2015; Lohmann \& Rebennack, 2016). These problems are typically concerned with the placement of power plants and thus utilize mixedinteger linear programming (Hobbs, 1995). In other streams of research, the focus is shifted to the reliability of the electricity supply (Billionnet et al., 2016), environmental metrics, or combinations in the form of multi-objective optimization (Antunes et al., 2004).

The previous works show considerable variety in the choice of decision variables. These primarily contribute to one (or more) of three areas, namely the dimensions of the power plant, its location, and the transmission network:

- Plant dimensions. For instance, Antunes et al. (2004) are concerned with power generation itself, such as the type and size of plants. Other research papers focus on the selection of the optimal generation mix. For instance, Billionnet et al. (2016) and Gupta et al. (2011) utilize standard solvers to find the optimal energy mix based on a mixed-integer linear problem for small hybrid stand-alone energy systems in remote villages and islands. Some works specifically incorporate demand-side management as this entails an interdependence with generation (Antunes et al., 2004). Furthermore, Parpas \& Webster (2014) employ Markov chains to model generation capacities on a long-term horizon.

- Location. A different stream of research is related to the placement of power plants. For instance, some studies evaluate the placement of specific power plant types. In this regard, Mustakerov \& Borissova (2010) address a mixed-integer non-linear discrete combinatorial problem to identify the optimal placement of wind turbines. In the discussion regarding where to locate distributed generation in electricity networks, some works refer to location planning (Kumar et al., 2016; Meneses de Quevedo \& Contreras, 2016; Thrampoulidis et al., 
2015). However, these frequently prevail to local distribution grids (e.g. as presented by the IEEE bus system). Research in this direction is relevant when deciding where to place a power source in a local neighborhood or how to design the surrounding grid, including converters and transformers.

- Transmission. In addition, multiple researchers present approaches to optimizing the transmission network design (Sherali \& Staschus, 1990; Villumsen \& Philpott, 2012). Qi et al. (2015) minimize transmission infrastructure costs for the delivery of wind power by adding energy storage systems close to wind farms in existing networks. For general network expansion planning, Mínguez \& García-Bertrand (2016) implement a computationally efficient decomposition approach. In contrast, Rossi et al. (2012) optimize the design of newly built radial power distribution networks by minimizing voltage drops between feeder and customer. Similarly, Kocuk et al. (2016) along with Villumsen \& Philpott (2012), evaluate how to improve network topologies by switching individual transmission lines on and off. With regards to transmission planning, Sherali \& Staschus (1990) as well as van der Weijde \& Hobbs (2012), present stochastic two-stage approaches to improve transmission line planning. In terms of operational costs, Hobbs \& Ji (1999) demonstrate how to estimate these by developing upper and lower cost bounds and employing stochastic programming to solve the problem.

It should be noted that a different stream of literature is concerned with planning local electricity networks (Marinakis et al., 2017); however, these local networks (i.e. so-called distribution networks) target only individual municipialities or districts, whereas we are concerned with large-scale (and often nationwide) transmission networks that connect individual distribution grids. Research in the context of distribution grids must address the specific physical constraints that arise in distribution grids (i. e. flow-based capacitated aborescence or other constraints due to low voltage settings) and thus experiment with other networks (e.g. bus systems). This thus differs considerably from our research setting (as transmission grids operate with high voltage and thus have a different set of physical constraints). Hence, we refer to outlets in the domain of electrical engineering such as IEEE for an detailed coverage of distribution grid planning (e.g. Baringo \& Conejo, 2012); however, their solution approaches are rarely applicable due to the different problem structure. 


\subsection{Integrated optimization of generation and transmission infrastructure}

Only few works actually focus on the combined problem of both generation and transmission (Conejo et al., 2016). For instance, Seddighi \& Ahmadi-Javid (2015) utilize a multistage stochastic programming model that facilitates the integrated planning of electricity generation and, simultaneously, optimizes the transmission network. However, this work solves only small instances with up to 17 locations and it deals merely with fixed costs without a variable component as it appears in the EGTP. Similarly, Pozo et al. (2013) present a mixed-integer linear programming problem that optimizes both generation and transmission planning in Chile. This again is applied to only relatively small-scale scenarios with fewer than 50 locations. Hence, the authors can solve the problem with CPLEX and are not concerned with the development of efficient optimization methods. The objective function of this problem also differs from the EGTP, as it includes an additional market clearing mechanism and sums over annual costs without disentangling investments into a fixed and variable component.

The above works are limited to small electricity systems, whereas we specifically indulge in the optimization of large-scale infrastructure for electricity generation and transmission with thousands of locations. This is especially challenging, as computation times increase dramatically. Since we are not aware of any research that holistically optimizes both power plants and the transmission network, we later need to develop different heuristics for solving our EGTP.

Relationship to plant location problems: Our EGTP is based on the physical properties of electricity, as well as energy systems, and thus differs in multiple respects from capacitated location problems such as SPLP and its variations. The SPLP determines the locations of industrial facilities by minimizing the sum of fixed costs, variable costs and transportation costs (Krarup \& Pruzan, 1983). The SPLP in its naïve form is based on a single plant type, while the EGTP specifically distinguishes a set $K$ of different types. Both allow only a single plant per location - an assumption that is relaxed in plant location problems with multiple facilities (Ghiani et al., 2002). Furthermore, the SPLP incorporates a linear cost function, while we explicitly enable non-linear cost behavior. In addition, SPLP builds upon a per-unit cost for transportation, whereas the transmission in the EGTP consists of two components, namely, fixed and variable costs. The EGTP additionally allows for the sharing of transmission lines; that is, electricity from different sources can use the same line for delivery. 


\section{The electricity generation and transmission problem (EGTP)}

This section introduces the assumptions underlying the EGTP and formalizes it mathematically.

\subsection{Problem description}

The EGTP problem draws upon a given number of locations. These can represent individual neighborhoods, districts, or whole cities. It is then based on the following assumptions:

- Cost minimization. The problem minimizes the combined costs for the infrastructure of power plants and the transmission network across all locations. This presents the objective function of the model. Depending on the choice of costs, these can represent purely the investments, or even include operational costs (i. e. when utilizing levelized costs of electricity). Furthermore, the individual costs of infrastructures depend on the specific location. In a practical setting, this allows us to reflect, for instance, additional earthworks, or that off-shore wind parks entail different investment costs than on-shore parks while generating the same volume of energy.

- Electricity demand. Each location entails a certain electricity demand that must be fulfilled. This can be achieved either by local electricity generation or by transmitting electricity from remote power plants.

- Transmission network. The costs of transmission lines scale linearly with their capacity, subject to a line-specific factor. The latter allows one to incorporate parameters that introduce costs which depend on the length of the transmission lines, as well as regional differences and power losses.

- Electricity generation. The problem incorporates different types of power plants. Illustrative examples are nuclear, coal, or wind power installations. The cost of each power plant depends on its energy source and the chosen generation capacity. In this regard, the unit cost of electricity is assumed to be non-linear with respect to the plant capacity, as suggested by research in the field of energy economics. A maximum of one plant can be placed at each location, consistent with the SPLP (Krarup \& Pruzan, 1983; Nara et al., 2001). This constraint is driven by practical considerations, which obviate the possibility of more than one generation unit per neighborhood or district. Conversely, the size of the plant is not limited, since, in practice, a plant often consists of several generation units. 
We have not defined what type of electricity demand and generation is considered, since our problem description is open to different objectives. Depending on the actual parameterization, one could use data concerning (a) the average demand/supply setting (i. e. as load in GW or, alternatively, as energy per time interval) in order to study a setting with baseline load or (b) a peak load setting (i. e. a high demand and low generation from variable renewable energy sources). The actual choice is left to potential users.

\subsection{Mathematical model}

This section formalizes the corresponding optimization problem. For this purpose, we provide an overview of the notation in Table 1. In the following, the optimization problem (and particularly the load therein) is specified in a general format for a predefined point in time, $t$, and, hence, without loss of generality, we omit the subscript for better readability. This is important to note as electricity production is often volatile due to variable renewable energy sources and, therefore, practitioners will address this by, for instance, performing the analysis with parameters that specify generation and transmission capacities in peak load scenarios.

Let $N$ denote the number of nodes of a (directed and weighted) graph. We refer to the corresponding nodes via indices $i, j=1, \ldots, N$. In practice, each node corresponds to, e.g., a city, and thus entails geographic coordinates such that we can compute the distance between two locations $i$ and $j$. Furthermore, each node comes with a given electricity demand $D_{i}$. This variable can refer to either the average demand per time slot or the peak demand, depending on whether the transmission network should be optimized against baseline or peak load.

The problem fulfills the electricity demand at each location by constructing power plants and transmission lines, for which we introduce decision variables in the following. ${ }^{1}$ We specifically assume different energy sources such that we build different infrastructure for power generation. For instance, we later report computational experiments utilizing generation types $K=\{$ solar, wind, biogas, brown coal, hard coal, gas $\}$. Furthermore, let the binary variable $e_{i}^{k}$ indicate whether a power plant of type $k$ (denoted as superscripts) is constructed at location $i$. Its capacity is given by $g_{i}^{k}$. Analogously, the binary variable $h_{i j}$ represents a binary flag if a transmission line with capacity $x_{i j}$ connects $i$ and $j$. We later utilize the binary variables to model the fixed costs, while the capacity relates to the variable costs.

\footnotetext{
${ }^{1}$ Note that we do not optimize power flows (i. e. energy in GWh) as is the usual case in distribution grids, since we focus on transmission grids and thus consider electricity load (measured in GW).
} 


\begin{tabular}{|c|c|c|}
\hline Symbol & Description & Unit/range \\
\hline \multicolumn{3}{|c|}{ GENERAL PROBLEM PARAMETERS } \\
\hline$N$ & Number of nodes & Positive integer \\
\hline$i, j$ & Indices of nodes (used as subscripts) & $i, j=1, \ldots, N$ \\
\hline$K$ & Set of all generation types & Set \\
\hline$k$ & Index for a specific generation type (used as superscripts) & $k \in K$ \\
\hline$D_{i}$ & Demand (e.g. average demand per time period or peak demand) at location $i$ & GW \\
\hline \multicolumn{3}{|c|}{ DECISION VARIABLES: ELECTRICITY GENERATION } \\
\hline$e_{i}^{k}$ & Binary variable; equals 1 if generation of type $k$ exists at location $i$ & $e_{i}^{k} \in\{0,1\}$ \\
\hline$g_{i}^{k}$ & Capacity of power plant at location $i$ of generation type $k$ & GW \\
\hline \multicolumn{3}{|c|}{ DECISION VARIABLES: ELECTRICITY TRANSMISSION } \\
\hline$h_{i j}$ & Binary variable; equals 1 if transmission between nodes $i$ and $j$ exists & $h_{i j} \in\{0,1\}$ \\
\hline$x_{i j}$ & Capacity of transmission line from node $i$ to $j$ & GW \\
\hline \multicolumn{3}{|c|}{ PARAMETERS: ELECTRICITY GENERATION } \\
\hline$\lambda_{i}^{k}$ & Fixed cost of electricity generation per unit at node $i$ of type $k$ & Monetary unit \\
\hline$\gamma_{i}^{k}$ & Variable cost of electricity generation at node $i$ of type $k$ & Monetary unit / GW \\
\hline$\kappa_{i}^{k}$ & Discount factor for non-linear generation cost at location $i$ of type $k$ & Dimensionless, $\kappa_{i}^{k}>0$ \\
\hline$M_{g}$ & Big M variable for generation capacity & $\mathrm{GW}$ \\
\hline \multicolumn{3}{|c|}{ PARAMETERS: ELECTRICITY TRANSMISSION } \\
\hline$\alpha_{i j}$ & Fixed cost of transmission line between $i$ and $j$ & Monetary unit \\
\hline$\beta_{i j}$ & Variable cost of transmission line between $i$ and $j$ & Monetary unit / GW \\
\hline$M_{x}$ & Big $M$ variable for transmission capacity & GW \\
\hline
\end{tabular}

Table 1: Notation in the EGTP formulation.

The optimization problem then determines both power plants located at the nodes and transmission lines between them in the form of weighted edges. It thereby minimizes the total costs that can cover investments either with or without operational costs depending on the choice of parameters. We further split the costs of both electricity generation and transmission into a component with fixed and variable costs, respectively. This yields the objective

$$
\min _{e_{i}^{k}, g_{i}^{k}, h_{i j}, x_{i j}} \sum_{i=1}^{N} \sum_{k \in K}\left[\lambda_{i}^{k} e_{i}^{k}+\gamma_{i}^{k}\left(g_{i}^{k}\right)^{\kappa_{i}^{k}}\right]+\sum_{i=1}^{N} \sum_{j=1}^{i}[\alpha_{i j} h_{i j}+\underbrace{\beta_{i j}}_{=\beta_{i j}^{\prime} \operatorname{dist}(i, j)}\left|x_{i j}-x_{j i}\right|],
$$

which we detail in the following. The first parenthesis refers to the total costs for the generation infrastructure and thus sums over all combinations of the $N$ nodes and the generation types in $K$. It thus comprises fixed costs $\lambda_{i}^{k}$ if the plant is present (i. e. $e_{i}^{k}$ equals one) and variable costs in the form of an exponentiation, where the corresponding exponent $\kappa_{i}^{k}$ governs the degree of non-linearity. Both costs are scaled with corresponding scalars $\lambda_{i}^{k}$ and $\gamma_{i}^{k}$, respectively. The second sum determines 
the costs of the transmission network. For this reason, it iterates exactly once over all transmission lines, which are given by pairs of $i$ and $j$ while skipping the symmetric counterpart. As before, the first part provides the fixed costs $\alpha_{i j}$ if the line is present (i. e. $h_{i j}$ equals one) and it is included only once for each line, regardless of the direction in which power is transmitted. The latter part refers to costs consisting of a line-specific parameter $\beta_{i j}$ (e.g. a multiple of the distance $\operatorname{dist}(i, j)$ between $i$ and $j$ ) and the net-energy transferred between the two locations. Note that the absolute value is not necessary in the case of non-linearity as this already makes simultaneous non-zero transmissions suboptimal; however, it turned out beneficial during our later implementation.

In addition, the above objective is subject to the following constraints:

$$
\begin{array}{rlrl}
D_{i} & =\sum_{k \in K} g_{i}^{k}+\sum_{j=1}^{N} x_{j i}-\sum_{j=1}^{N} x_{i j} & & i=1, \ldots, N \\
g_{i}^{k} & \geq 0 & i & =1, \ldots, N, \forall k \in K \\
x_{i j} & \geq 0 & i, j & =1, \ldots, N \\
x_{i i} & =0 & i & =1, \ldots, N \\
0 & \leq M_{g} e_{i}^{k}-g_{i}^{k} & i & =1, \ldots, N, \forall k \in K \\
0 & \leq M_{x} h_{i j}-x_{i j} & i & =1, \ldots, N \text { and } j=1, \ldots, i \\
0 & \leq M_{x} h_{j i}-x_{i j} & i & =1, \ldots, N \text { and } j=i+1, \ldots, N \\
\sum e_{i}^{k} & \leq 1 & i & =1, \ldots, N \\
k \in K & & i & =1, \ldots, N \\
h_{i j} & \in\{0,1\} & &
\end{array}
$$

Here, Equation (2) ensures that the demand at every node is satisfied by the power generation in that location together with the incoming volume of electricity, while subtracting the outgoing amount. Equations (3) and (4) require both the generation and transmission capacities to be nonnegative, while Equation (5) eliminates self-loops, i. e. that a transmission link starts and ends in the same node $i$. Equations (6) to (8) follow common techniques in location problems (Seddighi \& Ahmadi-Javid, 2015; Vasin \& Dolmatova, 2016) and utilize the Big M method. This introduces a capacity of zero (and thus zero variable cost) if the corresponding generation or transmission infrastructure is not constructed. Moreover, Equation (9) limits the number of power plants at each location to one. Lastly, Equations (10) and (11) ensure that the decision variables $e_{i}^{k}$ and $h_{i j}$ are binary, meaning that they define whether or not a power plant or transmission line is built. 
Note that no further physical constraints are necessary (different from meshed networks that are used in low-voltage distribution grids), since our objective is to model transmission networks where high-voltage current is transported.

Remark 1. For $\kappa_{i}^{k} \geq 1$ for all $i$ and $k$, the EGTP is a convex MINLP problem.

Remark 2. The EGTP is NP-hard.

The above formulation of the EGTP presents a MINLP problem, since it incorporates binary decision variables and a non-linear expression in the objective. This problem is NP-hard, since it can be shown that NP-hard SPLP presents a sub-problem as follows. Without loss of generality, we assume the same number of locations for generation and demand. We further allow only one generation type, given by $|K|=1$, remove the non-linearity via $\kappa_{i}^{k}=1$, and omit investment costs for transmission by setting $\alpha_{i j}=0$. This yields a sub-problem of EGTP that is equivalent to the definition of the SPLP (Krarup \& Pruzan, 1983).

\subsection{Example}

This section presents an illustrative example concerning our EGTP and compares different, feasible solutions. Our example consists of $N=3$ locations given by points $P_{1}=(0,1), P_{2}=(4,0)$ and $P_{3}=(0,4)$ on a grid, as visualized in Figure 1 . Their electricity demand amounts to $D_{1}=0.5$, $D_{2}=1.0$ and $D_{3}=1.5$, respectively. For simplicity, we further assume a single generation type with the same generation cost in each node. All remaining costs of generation and transmission are specified in Table 2. Here, the scalars $\beta_{i j}$ for the variable transmission costs are set to the $L_{2}$-distance between $i$ and $j$.

\begin{tabular}{ll}
\hline Generation costs & $\lambda=3, \gamma=4, \kappa=0.8$ \\
Transmission costs & $\alpha=0.5, \beta_{12}=2.24, \beta_{13}=3, \beta_{23}=4$ \\
\hline
\end{tabular}

Table 2: Illustrative parameters concerning the generation and transmission infrastructure in our example.

We proceed by illustrating three feasible solutions (cf. Figure 1) as follows:

1. A distributed solution positions the electricity generation in each node such that it equals the local demand. As a result, each node entails its own power plant, resulting in generation costs of $\left(3+4 \cdot 0.5^{0.8}\right)+\left(3+4 \cdot 1.0^{0.8}\right)+\left(3+4 \cdot 1.5^{0.8}\right)=20.83$ and zero transmission costs.

2. A centralized solution fulfills the electricity demand by transmitting electricity from a single power plant. This increases transmission costs but can potentially exploit lower generation costs in other locations. In our example, we decided to assign the central power plant to the 

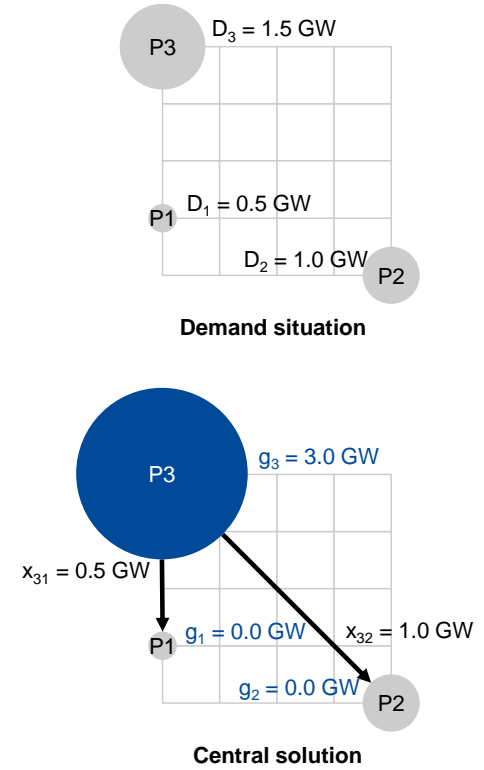

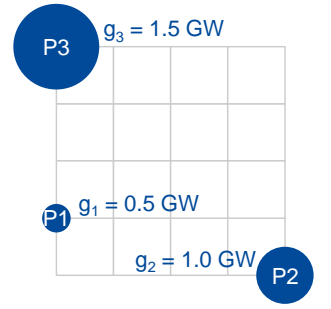

Distributed solution

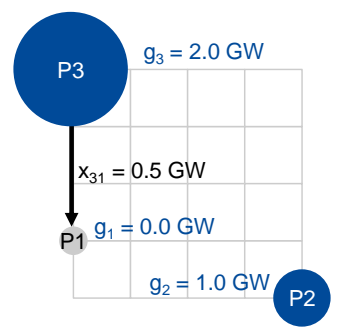

Optimal solution
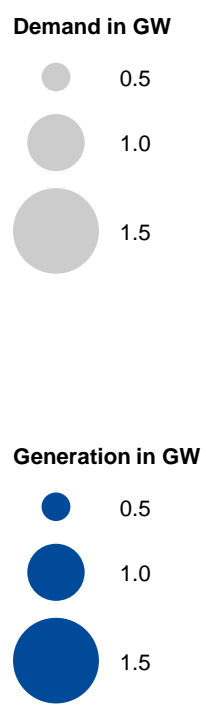

Figure 1: Illustration of a generic location optimization task and various solutions.

node with the highest demand in order to reduce the variable factor of transmission costs. Here, the corresponding node is given by $P_{3}$ with a demand of $D_{3}=3$. This necessitates two transmission lines $P_{3} \rightarrow P_{1}$ and $P_{3} \rightarrow P_{2}$ with capacities of $x_{31}=0.5$ and $x_{32}=1.0$, respectively. This results in generation costs of 12.63 , transmission costs of 8.16, and 20.79 in total.

3. The optimal solution, which minimizes the total costs, is visualized in Figure 1. Its costs amount to only 18.96, which it achieves by removing the expensive transmission line $P_{3} \rightarrow P_{2}$ from the centralized solution and, instead, creates a separate power plant in $P_{2}$.

\section{Optimization methods}

The EGTP can be approached by MINLP solvers such as the branch-and-reduce. These, for instance, exploit the convex shape of the objective function or circumvent non-linearities through linearization. However, our experiments later in Section 5 demonstrate that even moderate instances exceed practical runtime limits $(N \geq 25)$. This necessitates the development of alternative optimization methods and thus, in this section, we develop different heuristics, which generate feasible solutions by exploiting the geometric structure of the problem:

Construction heuristics. Our centralized heuristic bundles all electricity generation into a single power plant which supplies all other nodes by transmitting electricity. The distributed heuristic, in contrast, creates power plants in each location such that the local demand is fulfilled 
without power transmission. A greedy construction heuristic combines both approaches by establishing smaller regions with centralized generation in each in order to achieve a trade-off between centralized and distributed generation.

Improvement heuristics. Our improvement heuristics build upon the feasible solutions from the construction heuristics and then perform a reduced variable neighborhood search.

GRASP metaheuristic. The previous construction and improvement heuristics can be combined in a GRASP metaheuristic.

We also experimented with other (heuristic) solution techniques; however, these are often not directly applicable due to the inherent structure of our optimization problem. Linearization is later implemented as part of our exact solvers, though without obtaining performance improvements. A possible explanation is that the complexity of our problem is largely owed to the binary decision variables (and not the non-linear objective function). Further, linearization with subsequent application of Benders decomposition (cf. e.g. Baringo \& Conejo, 2012) would be an interesting option, yet such a decomposition is impeded by the term $x_{i j}$ in the objective function. Finally, we experimented with a warm-start procedure where an exact solver was initialized based on the heuristics but the nature of the employed exact solvers could not benefit from this; hence, we omitted the results.

\subsection{Construction heuristics}

\subsubsection{Centralized generation}

The centralized construction heuristic chooses a single generation plant that supplies all remaining locations. The location of this single plant can be picked arbitrarily; however, our experiments identified the node with the highest demand as a particularly beneficial choice, since it diminishes the need for transmission capabilities and thus reduces transmission costs. As a result, the centralized heuristic achieves fairly low generation costs due to economies of scale, but entails high transmission costs. It often exhibits a sound performance in cases where transmission costs are low relative to the generation costs (e.g. due to short distances between nodes). Algorithm 1 provides the pseudocode. The loop has a runtime of $\Theta(N|K|)$ and, additionally, the matrix initializations in Step 2 are in $\Theta\left(N^{2}+N|K|\right)$. 


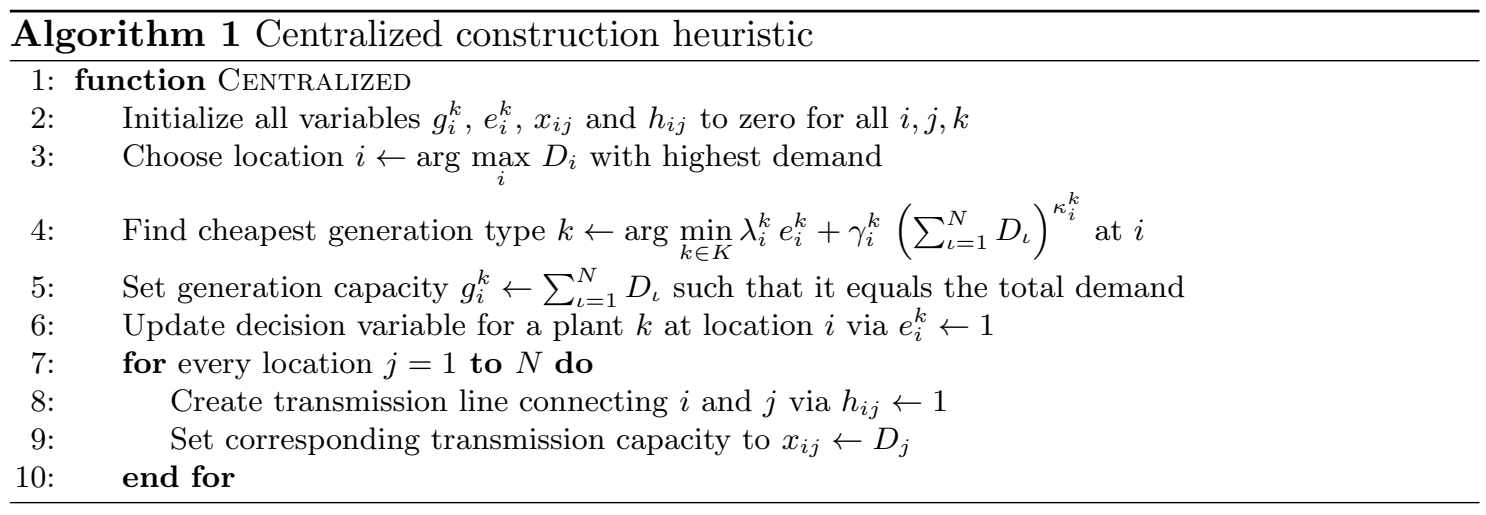

\subsubsection{Distributed generation}

This distributed heuristic picks the cheapest electricity generation type for each location and sets its production to the demand of the node. Hence, it benefits from zero transmission costs. Algorithm 2 presents the pseudocode, which has a runtime of $\Theta(N|K|)$ due to Step 4 and, additionally, $\Theta\left(N^{2}+N|K|\right)$ for the matrix initializations in Step 2.

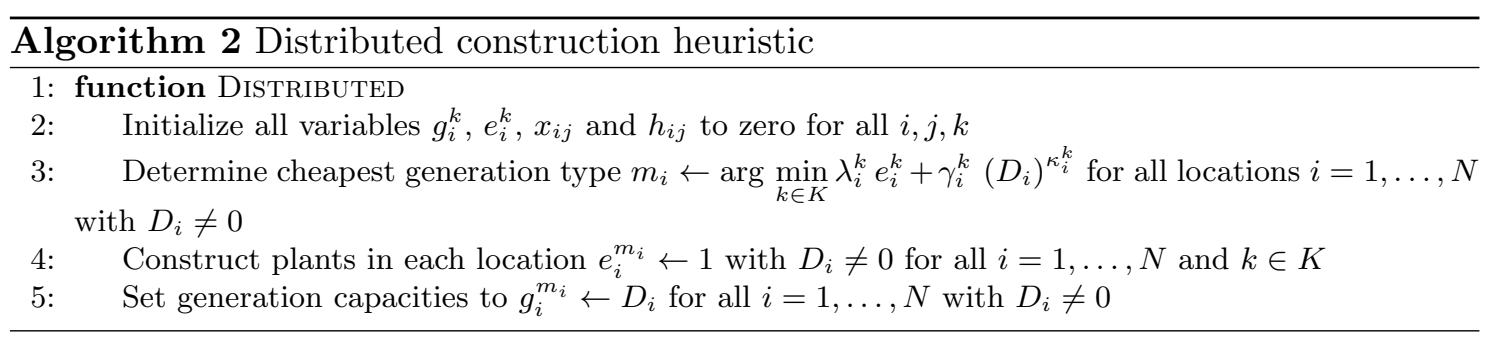

\subsubsection{Greedy heuristic}

The greedy heuristic constructs local areas, each with its own centralized generation source, while the areas are not necessarily connected via transmission lines. For this purpose, all nodes are sorted in decreasing order of generation costs necessary to meet their own demand $D_{i}$. Afterwards, we iterate over all nodes in that order and build transmission lines to all neighboring nodes for which transmission is cheaper than local generation with respect to fulfilling their electricity demand. We report the corresponding pseudocode in Algorithm 3, which also entails a randomization procedure that is later used in the GRASP metaheuristic, which relies in turn on the greedy construction heuristic. The runtime is in $O\left(N^{2}|K|\right)$, plus $\Theta\left(N^{2}+N|K|\right)$ for the matrix initializations in Step 2. 


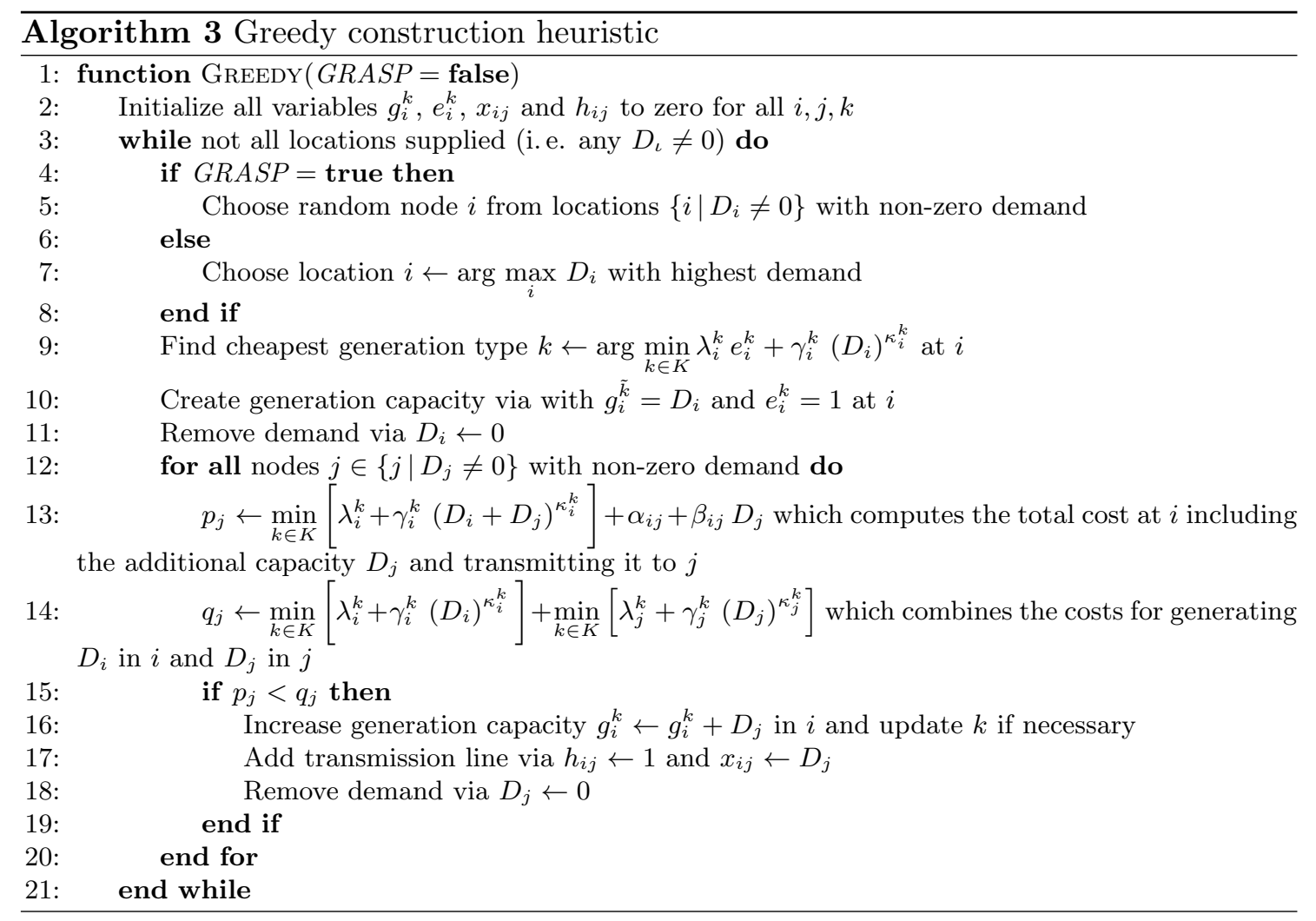

\subsection{Improvement heuristic}

Our improvement heuristic utilizes a reduced variable neighborhood search (Hansen et al., 2010) in order to test whether local modifications improve the current solution. For this purpose, the heuristic alters the current solution to include a greater degree of either centralized or distributed electricity generation and then compares the outcomes to the initial solution. If this results in a decrease of the objective function, it continues its search on the basis of the modified solution.

Algorithm 4 formalizes the above search process given an initial solution $\mathcal{S}$ and a parameter $\eta$ controlling the number of iterations of the local search. The search then repeatedly draws randomly selected locations $i$ and determines the nearest neighbor $j$. It then applies the following modifications to the pair $i$ and $j$ :

- If $i$ or $j$ produce electricity, we validate whether we improve the solution by relocating power generation to either $i$ or $j$. This modification seeks improvements from further centralization. The relocation is performed by Algorithm 5 (ADDTRANSMISsion), which relocates power generation from $i$ to $j$ and adds the necessary transmission infrastructure. Here, we test two possibilities as we can move power generation either from $i$ to $j$, or from $j$ to $i$. The newly created transmission lines make the solution more centralized. 
- In contrast, Algorithm 6 presents the function RemoveTransmission, which removes any transmission capacity between the two locations $i$ and $j$. It thus updates the generation infrastructure at that location and then removes the transmission line, thereby resulting in a more distributed solution. This leads to an increase or decrease in power generation at both ends of the transmission line.

The algorithm repeats these neighborhood modifications, such that the degree of centralization varies continuously.
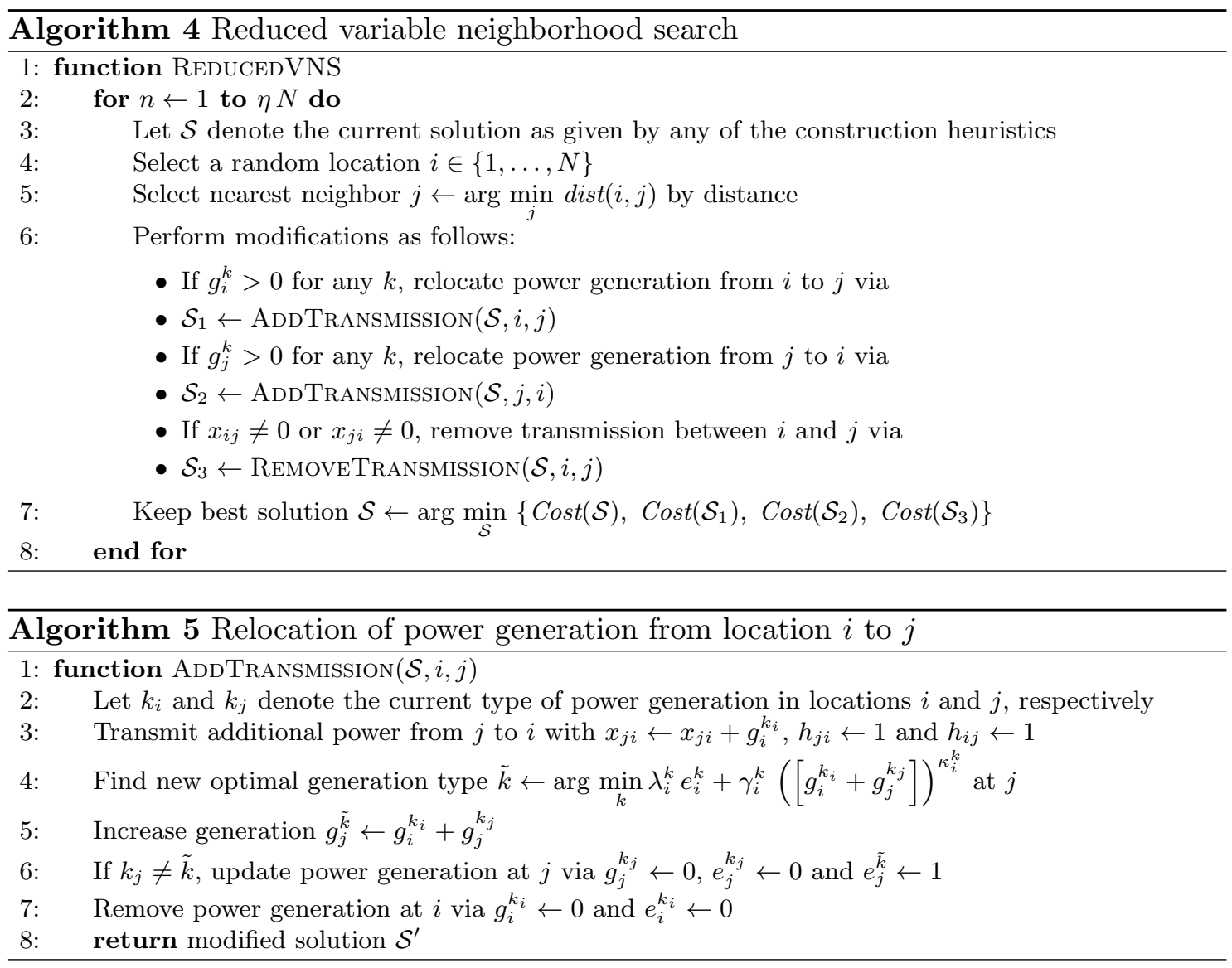


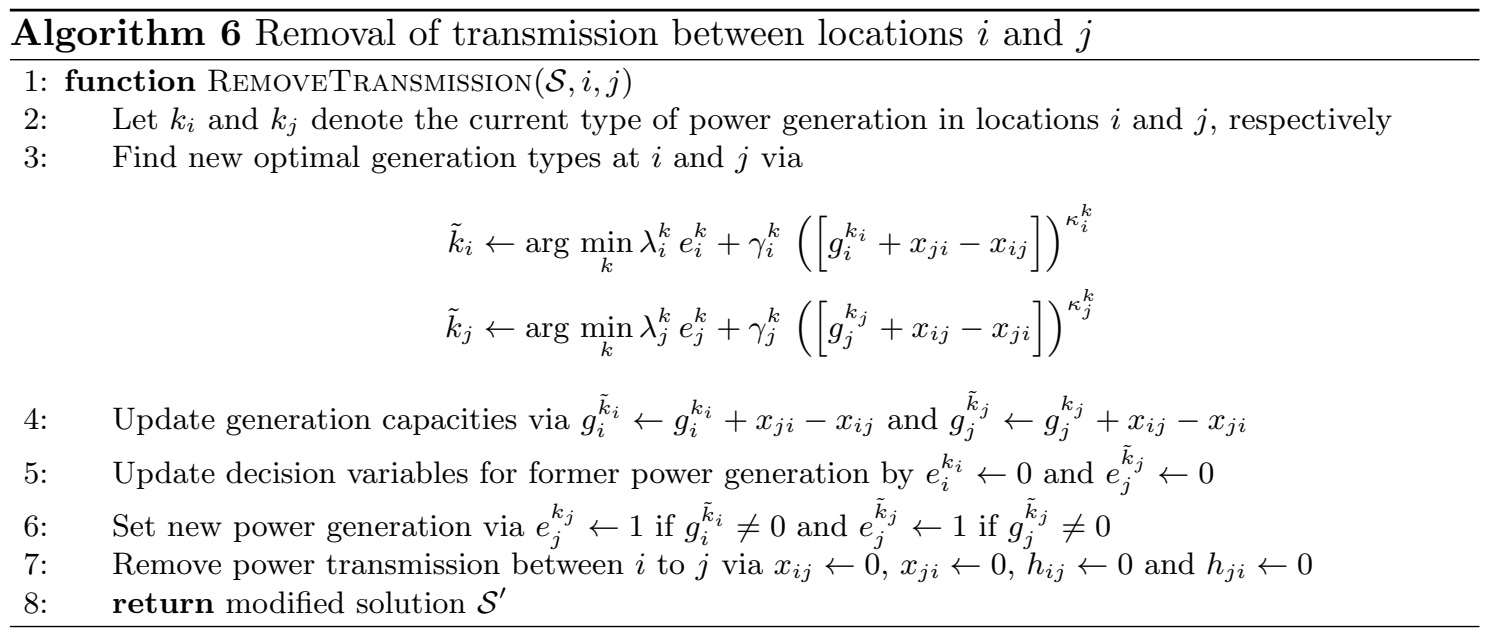

\subsection{GRASP metaheuristic}

The performance of improvement heuristics largely depends on the starting point of their search. A potentially better alternative is represented by the greedy randomized adaptive search procedure, which provides a multi-start metaheuristics as follows (Feo \& Resende, 1995; Resende \& Ribeiro, 2010). It utilizes a construction heuristic with randomization in order to generate a collection of initial, feasible solutions. Based on these, GRASP then applies improvement heuristics to perform a local search.

In our case, the GRASP implementation draws upon the greedy construction heuristic from Section 4.1, which is modified to include an additional randomization. This then serves as an input to the neighborhood search, which is similarly extended by a randomization component. For this reason, the GRASP procedure (see Algorithm 7) utilizes a restricted candidate list from which it picks neighbors. The variable $\Phi$ denotes the number of GRASP iterations and $\rho$, as well as $\Psi$, are additional parameters.

The GRASP routine utilizes a modified improvement heuristic REDUCEDVNS GRASP $_{\text {that in- }}$ corporates additional randomization. Instead of selecting the nearest neighbor $j$, as the original REDUCEDVNS heuristic does, it randomly chooses a location within a certain proximity, to which it then applies the improvements. This is given by

$$
j \in\left\{j \mid \operatorname{dist}(i, j) \leq \min _{\tau} \operatorname{dist}(i, \tau)+\rho\left[\max _{\tau} \operatorname{dist}(i, \tau)-\min _{\tau} \operatorname{dist}(i, \tau)\right]\right\},
$$


where the proximity is controlled by the parameter $\rho$.

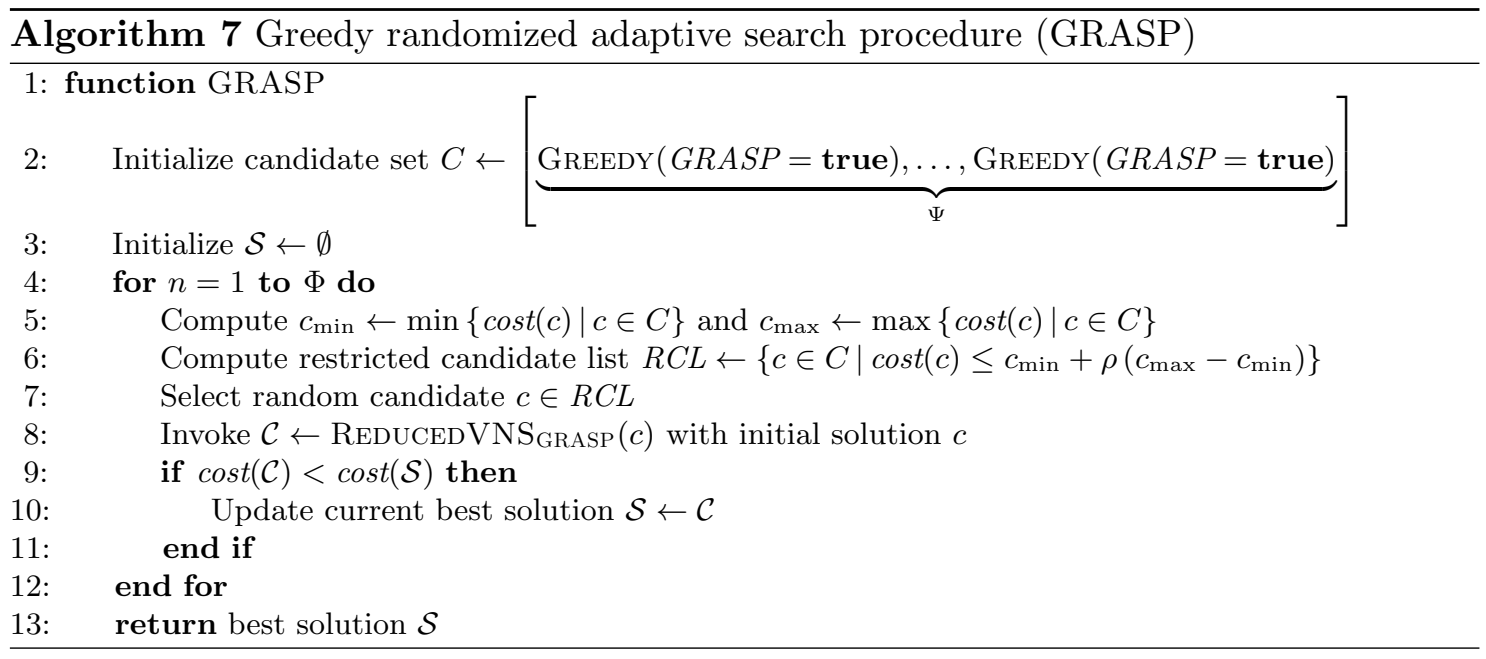

\subsection{Baseline: branch-and-reduce}

We choose the branch-and-reduce method as the baseline in evaluating our heuristics as it can find the global optimum of a MINLP. This algorithm defines a deterministic branch-and-bound search that exploits convexity and generates corresponding polyhedral cutting planes (Tawarmalani \& Sahinidis, 2005). For reasons of comparability, we utilize the implementation inside the General Algebraic Modeling System (GAMS) given by the BARON solver. As part of our sensitivity analysis, we also experiment with all other solvers for MINLP shipped as part of GAMS, namely, ANTIGONE, AlphaECP, SBB, BARON and DICOPT. The corresponding results are detailed in the online appendix, suggesting that BARON performs best overall. For performance reasons, we incorporate additional constraints in the GAMS solvers that leave the practical solution unchanged but reduce the search space. These are also documented in the online appendix.

We point further towards the following important characteristics:

1. Given $\kappa \geq 1$, our problem is convex and, hence, this fact can be leveraged by MINLP solvers. To this end, the convexity of our problem is actually leveraged by AlphaECP, DICOPT and SBB, which we considered for this purpose. However, contrary from our expectations, we observed performance improvements when choosing a different solver that is not capable of targeting a convex MINLP; namely, the BARON solver.

2. It could be an efficient strategy to apply linearization to our objective function. Such linearizations are implemented in ANTIGONE and BARON by default. Nevertheless, we see that linearization is not capable of reducing computation times down a tolerable limit, since the 
large number of binary variables remains. Therefore, the problem complexity stems more from the binary decision variables as from the non-linear objective function. This also explains why our proposed heuristics are numerically beneficial: they exploit the problem structure based on which they reduce the complexity of the grid layout.

\section{Computational experiments}

This section compares the performance of the baseline solver with our heuristics for instances in the range of 5 to 200 nodes. This helps us to understand the characteristics of the optimization methods, while actual problems are likely to span $N \geq 1,000$ nodes in practice, such as our realworld case study introduced in Section 6. Our computational experiments demonstrate that the branch-and-reduce solver exceeds our runtime limit of two hours, even for small cases of $N \geq 25$. This threshold was necessary due to the overall number of instances that we computed and the resulting total demand of computation demand which was in the order of 10,000 computing hours. In contrast, our proposed heuristics enable us to find efficient solutions within seconds. The runtime limits were omitted later in the case study that is considerably larger in size and, hence, individual solvers run up 460 hours.

All results originate from experiments with an Intel Xeon E5-2680 CPU at $2.7 \mathrm{GHz}$ and $16 \mathrm{~GB}$ of RAM. The heuristics are implemented in Python 2.7. For each setup, we average the results from 10 random instances of the same scenario.

\subsection{Data generation}

We design experiments that compare two common scenarios - a dense and a sparse setting in order to reflect the differences in energy systems between regions and countries. We further incorporate actual costs for electricity generation and transmission infrastructure. We make no differences between whether we optimize against baseline or peak load (hence, we refer to simply as demand and, without loss of generality, assume that the analysis is for an average of the day), since the purpose of the following experiments is to evaluate the ability of the optimization methods in solving the overall problem definition.

\subsubsection{Scenarios}

Our scenarios reflect the common differences between countries, which vary in their population density, their demand structure and, especially, in the average distances between adjacent cities. We thus consider a dense scenario that assumes a region with a largely urban environment, high 
population density, and short distances between cities. Such properties correspond with countries such as Great Britain, Germany, or Japan. As a comparison, we also study a sparse setting, which features a rural landscape with relatively long distances between cities. Illustrative examples include the United States, Canada, or Australia. Both scenarios favor solutions with different geometric characteristics. In the case of a dense scenario, a centralized solution might be financially preferable, while a distributed solution is likely to be more efficient in the sparse setting, where transmission costs are higher. Our model definition makes no specific assumptions whether the demand $D_{i}$, $i=1, \ldots, N$ is parameterized based on the average load or the peak load, since the analysis can be easily repeated with different values. Hence, we follow earlier literature (Billionnet et al., 2016; Zhan et al., 2017) and demonstrate our EGTP based on the (averaged) historic grid load.

For each scenario, we construct problem instances in which we place $N \in\{5,10,25,100,200\}$ nodes randomly on a grid. The $x$ and $y$ coordinates of the nodes are sampled from a uniform

distribution $\mathcal{U}\left(0, c_{\text {scale }} \sqrt{N}\right)$, where the parameter $c_{\text {scale }}$ controls how closely individual nodes are placed. In the following, we utilize $c_{\text {scale }}=25$ for the dense and $c_{\text {scale }}=125$ for the sparse scenario. The demand at each location is sampled from a mixture of two normal distributions: $80 \%$ of the values stem from $\mathcal{N}(0.02,0.008)$ and $20 \%$ from $\mathcal{N}(0.02,0.8)$ in order to reflect both provincial towns and metropolises. We subsequently compute the absolute value of the demand in order to eliminate negative numbers. This results in a demand distribution that is evident in many countries where populations are split between a few large cities and many small municipalities.

\subsubsection{Generation cost}

Our experiments comprise of the following types of power generation: solar, wind, biogas, brown coal, hard coal, and gas. These represent the main types of power generation that are currently in use, especially in developed countries such as Germany. We then set the parameters for the costs of electricity generation to the actual levelized cost of electricity (Bemis \& DoAngelis, 1990). These values are common in related research in the field of energy systems, since they incorporate not only upfront investments, but also generation-specific discount rates, facility lifespan, and operational costs (transport, maintenance, labor, fuel costs, carbon price, plant efficiency). In this paper, we sample all cost-related parameters from normal distributions, as specified in Table 3, in order to account for local variation, such as land cost, transportation needs of fossil fuels, or different efficiencies of renewable energy sources. We use levelized costs of electricity that are comparable to expert estimates for Germany and similar to figures found in related works (e.g. Stolzenberger, 2015). We observe that the variable costs are below the initial investments, which introduces 
economies of scale. In addition, we assume a non-linear cost function, which is consistent with empirical findings as motivated earlier. As a result, the exponent $\kappa>1$ penalizes power plants of growing size and thereby adheres to physical limitations. Our experiments later confirm that this parameter choice yields realistic conditions.

\begin{tabular}{|c|c|c|c|c|c|c|}
\hline \multirow[t]{2}{*}{ Generation type } & \multicolumn{2}{|c|}{ Fixed generation cost $\lambda$} & \multicolumn{2}{|c|}{ Variable generation cost (per capacity) $\gamma$} & \multicolumn{2}{|c|}{ Exponent $\kappa$} \\
\hline & Mean & Std. dev. & Mean & Std. dev. & Mean & Std. dev. \\
\hline Solar & 1.0 & 0.4 & 0.097 & 0.039 & 1.027 & 0.054 \\
\hline Wind & 310.8 & 174.5 & 0.054 & 0.069 & 1.138 & 0.158 \\
\hline Biogas & 21.9 & 10.0 & 0.141 & 0.080 & 1.059 & 0.078 \\
\hline Brown coal & $11,375.0$ & 3750.0 & 0.036 & 0.015 & 1.022 & 0.026 \\
\hline Hard coal & $14,300.0$ & 3400.0 & 0.056 & 0.017 & 1.019 & 0.019 \\
\hline Gas & $10,812.5$ & 2875.0 & 0.068 & 0.023 & 1.021 & 0.022 \\
\hline
\end{tabular}

Table 3: The parameters in this table specify the normal distributions from which we sample the costs for power generation. Here, we specifically utilize the levelized costs of electricity (in $€ / \mathrm{kWh}$ ), as they incorporate both investment and operational costs. Note that both coal and gas obtain attain fairly high fixed generation costs. However, when taking the size of the different plants and the variable generation cost into account, the resulting costs resemble the overall levelized costs of electricity as in (e. g. Stolzenberger, 2015).

\subsubsection{Transmission costs}

Similar to the generation costs, we introduce line-specific transmission costs and, for this purpose, sample transmission costs in $€$ per $\mathrm{kWh}$ and $\mathrm{km}$ from a normal distribution, as specified in Table 4. Notably, a variable transmission costs $\beta^{\prime}$ is sampled (in monetary units per capacity and distance) and afterwards multiplied by the distance between two locations in order to give $\beta$ (in monetary units per capacity). In a subsequent step, these values are multiplied by the length of the transmission line. This approach and the corresponding values reflect suggestions from previous research (e.g. Delucchi \& Jacobson, 2011).

\begin{tabular}{lcccc}
\hline \multicolumn{2}{l}{ Fixed transmission $\operatorname{costs} \alpha$} & & \multicolumn{2}{l}{ Variable transmission costs (per capacity and distance) $\beta^{\prime}$} \\
\cline { 1 - 1 } \cline { 1 - 1 } Mean & Std. dev. & & Mean & Std. dev. \\
\hline 0.088 & 0.059 & & 0.000699 & 0.000879 \\
\hline
\end{tabular}

Table 4: The parameters specify the normal distributions from which we sample the costs of power transmission (in $€$ per $\mathrm{kWh}$ and $\mathrm{km}$ ). After sampling, the values of $\beta^{\prime}$ are multiplied with the distance between two locations $i$ and $j$ in order to give the actual $\beta$.

\subsection{Solver parameters}

In the improvement heuristic, the maximum search iteration parameter $\eta$ controls the extent of tested improvements; a higher value increases execution time but is more likely to identify solutions 
with lower costs. We experimented with a variety of values as detailed in the online appendix and, accordingly, decided upon a maximum search iteration parameter $\eta=2$, which is additionally multiplied by the grid size $N$. Then the total number of tested improvements is $\eta N$. In the case of GRASP, we follow Resende \& Ribeiro (2010) and set the proximity $\rho$ of restricted candidates to 0.2. This provides a reasonable trade-off between runtime and the extent of exploration. In addition, the GRASP iteration number $\Phi$ specifies how often GRASP invokes the greedy construction heuristic. Since we observe only small variations in the greedy solutions, we utilize $\Phi=5$ and $\Psi=5$ GRASP iterations in the following.

\subsection{Results from the dense scenario}

Table 5 compares the optimization methods in the dense scenario. Our baseline, branch-andreduce, solves small instances $(N=5$ and 10) optimally, while its execution time quickly explodes and exceeds our runtime limit already for $N \geq 25$. For some instances, we can still report the last iteration of branch-and-reduce, yet it presents merely an approximation of the global optimum. This necessitates alternative optimization methods for approaching the EGTP.

Both neighborhood heuristics lower costs further as compared to the construction heuristics, but slightly increase the computational time. Here we observe the following patterns: the neighborhood search reduces the expenditures for electricity generation beyond the solutions found by the construction heuristics. Overall, both variants find solutions where the number of power plants is close to $N / 2$ (similar to the greedy heuristic). For large problems with $N=100$ and 200 , the combination of distributed construction heuristic and neighborhood search obtains the best solutions across all optimization methods.

The GRASP algorithm usually yields solutions with costs below the other heuristics in the small instances. Its computation time exceeds the runtime of the other heuristics, but we can still compute instances with $N=200$ nodes in reasonable time, i. e. $13.2 \mathrm{~min}$. On average, the runtimes are roughly five times longer than those of the neighborhood search, which matches our choice of $\Phi=5$.

In summary, even though branch-and-reduce might be able to identify the global optimum in small instances, its usage is infeasible in practical applications due to the excessive runtime. Here the experiments identify our heuristics as viable alternatives, since several of them find solutions whose objective function is close to the branch-and-reduce method, but require only a fraction of the computational resources. 


\begin{tabular}{|c|c|c|c|c|c|c|c|c|}
\hline$N$ & Solver & $\begin{array}{l}\text { Total } \\
\text { time }(s)\end{array}$ & $\begin{array}{l}\text { Total cost } \\
(\mathrm{mn} \text { EUR/h) }\end{array}$ & $\begin{array}{l}\text { Variance of total } \\
\text { cost across runs }\end{array}$ & $\begin{array}{l}\text { Generation } \\
\text { cost (mn EUR/h) }\end{array}$ & $\begin{array}{l}\text { Transmission } \\
\text { cost (mn EUR/h) }\end{array}$ & $\begin{array}{c}\text { Relative cost } \\
(=\text { total cost / } \\
\left.\text { overall demand } \sum D_{i}\right)\end{array}$ & $\begin{array}{l}\text { Avg. plant } \\
\text { count }\end{array}$ \\
\hline \multirow{7}{*}{5} & Centralized heuristic & 0 & 0.054 & 0.003 & 0.052 & 0.002 & 0.058 & 1.0 \\
\hline & Distributed heuristic & 0 & 0.057 & 0.003 & 0.057 & 0.000 & 0.065 & 5.0 \\
\hline & Greedy heuristic & 0 & 0.054 & 0.003 & 0.053 & 0.001 & 0.058 & 3.0 \\
\hline & Neighborhood search (with centralized heuristic) & 1 & 0.038 & 0.001 & 0.030 & 0.007 & 0.046 & 2.2 \\
\hline & Neighborhood search (with distributed heuristic) & 1 & 0.038 & 0.001 & 0.031 & 0.006 & 0.045 & 2.8 \\
\hline & GRASP & 5 & 0.031 & 0.000 & 0.021 & 0.010 & 0.038 & 2.0 \\
\hline & Branch-and-reduce (GAMS/BARON) & 26 & 0.028 & 0.000 & 0.012 & 0.016 & 0.034 & 2.3 \\
\hline \multirow{7}{*}{10} & Centralized heuristic & 1 & 0.088 & 0.004 & 0.069 & 0.019 & 0.062 & 1.0 \\
\hline & Distributed heuristic & 0 & 0.080 & 0.001 & 0.080 & 0.000 & 0.061 & 10.0 \\
\hline & Greedy heuristic & 1 & 0.072 & 0.002 & 0.066 & 0.005 & 0.053 & 5.0 \\
\hline & Neighborhood search (with centralized heuristic) & 2 & 0.054 & 0.001 & 0.040 & 0.014 & 0.043 & 3.0 \\
\hline & Neighborhood search (with distributed heuristic) & 2 & 0.051 & 0.001 & 0.044 & 0.007 & 0.043 & 5.7 \\
\hline & GRASP & 11 & 0.035 & 0.000 & 0.023 & 0.012 & 0.029 & 3.6 \\
\hline & Branch-and-reduce (GAMS/BARON) & 83 & 0.029 & 0.000 & 0.016 & 0.013 & 0.024 & 3.9 \\
\hline \multirow{7}{*}{25} & Centralized heuristic & 1 & 0.333 & 0.016 & 0.202 & 0.131 & 0.091 & 1.0 \\
\hline & Distributed heuristic & 1 & 0.218 & 0.004 & 0.218 & 0.000 & 0.059 & 25.0 \\
\hline & Greedy heuristic & 2 & 0.191 & 0.004 & 0.169 & 0.023 & 0.052 & 13.0 \\
\hline & Neighborhood search (with centralized heuristic) & 5 & 0.149 & 0.006 & 0.079 & 0.070 & 0.038 & 8.2 \\
\hline & Neighborhood search (with distributed heuristic) & 5 & 0.129 & 0.001 & 0.100 & 0.029 & 0.035 & 13.9 \\
\hline & GRASP & 32 & 0.125 & 0.002 & 0.080 & 0.045 & 0.033 & 9.8 \\
\hline & Branch-and-reduce (GAMS/BARON) & 7239 & $0.078^{\dagger}$ & $0.001^{\dagger}$ & $0.037^{\dagger}$ & $0.041^{\dagger}$ & $0.020^{\dagger}$ & $11.4^{\dagger}$ \\
\hline \multirow{7}{*}{100} & Centralized heuristic & 16 & 1.944 & 0.438 & 0.549 & 1.395 & 0.127 & 1.0 \\
\hline & Distributed heuristic & 14 & 0.783 & 0.006 & 0.783 & 0.000 & 0.051 & 100.0 \\
\hline & Greedy heuristic & 14 & 0.716 & 0.009 & 0.556 & 0.160 & 0.047 & 50.0 \\
\hline & Neighborhood search (with centralized heuristic) & 28 & 0.783 & 0.029 & 0.521 & 0.262 & 0.051 & 44.0 \\
\hline & Neighborhood search (with distributed heuristic) & 44 & 0.575 & 0.006 & 0.513 & 0.062 & 0.038 & 56.6 \\
\hline & GRASP & 236 & 0.621 & 0.004 & 0.362 & 0.259 & 0.041 & 40.4 \\
\hline & Branch-and-reduce (GAMS/BARON) & 7351 & $0.967^{\dagger}$ & $0.244^{\dagger}$ & $0.854^{\dagger}$ & $0.113^{\dagger}$ & $0.063^{\dagger}$ & $72.4^{\dagger}$ \\
\hline \multirow{7}{*}{200} & Centralized heuristic & 58 & 6.408 & 1.974 & 1.178 & 5.230 & 0.220 & 1.0 \\
\hline & Distributed heuristic & 55 & 1.537 & 0.028 & 1.537 & 0.000 & 0.053 & 200.0 \\
\hline & Greedy heuristic & 55 & 1.486 & 0.046 & 1.001 & 0.485 & 0.051 & 100.0 \\
\hline & Neighborhood search (with centralized heuristic) & 96 & 1.951 & 0.423 & 0.899 & 1.052 & 0.067 & 90.5 \\
\hline & Neighborhood search (with distributed heuristic) & 195 & 1.093 & 0.019 & 0.949 & 0.143 & 0.037 & 110.5 \\
\hline & GRASP & 791 & 1.393 & 0.017 & 0.758 & 0.635 & 0.048 & 86.7 \\
\hline & Branch-and-reduce (GAMS/BARON) & - & - & - & - & - & - & - \\
\hline
\end{tabular}

Table 5: These experiments compare the runtime and the characteristics of the solutions from the different optimization methods in the dense scenario with $c_{\text {scale }}=25$. Results are averaged across 10 random instances. The variance of the retrieved objective (i. e. the total costs) across 10 runs is reported in order demonstrate the stability with which the different approaches generate solutions. The column with relative costs denotes the ratio of total cost to overall demand. It is thus independent of $N$ in order to compare the solutions across different instances of $N$. The legend additionally indicates experiments where solvers exceed our runtime limit of $2 \mathrm{~h}$ (i. e. 7,200s). The best solution approach for each $N$ is highlighted in bold. 


\subsection{Results from the sparse scenario}

Table 6 compares the optimization methods and the solutions in the sparse scenario. Again, the baseline solver, branch-and-reduce, finds optimal solutions for small scenarios with $N=5$ and $N=10$. Similarly to the dense scenario, its execution time exceeds our runtime limit already for $N=25$.

Analogously to the dense scenario, the centralized and distributed construction heuristics find solutions with 1 and $N$ plants, respectively. The distributed construction heuristic does not require any transmission and, thus, distances between locations may be ignored. Consequently, it achieves the same cost in the dense and sparse scenarios. In contrast, the centralized construction heuristic results in more expensive solutions in the sparse scenario due to longer transmission lines.

The increased distances between locations also result in a diminished performance of the neighborhood search with centralized heuristic. This heuristic yields in solutions which are even more expensive than those in the dense scenario. Again, the neighborhood search with distributed heuristic achieves the lowest cost of all optimization methods for $N \geq 25$.

Our GRASP implementation yields the lowest-cost solutions in the $N=5$ and 10 scenarios. In the other scenarios, with higher $N$, it does not outperform the neighborhood heuristic and also takes significantly longer. However, in contrast to branch-and-reduce, execution times are still feasible for $N=200$.

Branch-and-reduce identifies optimal solutions in the small scenarios with $N=5$ and $N=10$. However, compared to the dense scenario, these solutions are more expensive (costs almost doubles) and make use of many more plants. Thus, in the sparse scenario, branch-and-reduce finds solutions which are closer to a distributed solution, whereas the optimal solutions to the dense scenario resemble relatively centralized arrangements.

\subsection{Comparison of dense and sparse scenario}

Tables 5 and 6 also reveal interesting characteristics in which the solutions differ across both scenarios. The average total costs in the sparse scenario generally exceed those of the dense scenario, since they require higher investments in transmission infrastructure or, alternatively, additional plants with accumulated fixed costs. This difference becomes especially apparent when focusing on the relative costs. These span the interval from $€ 0.02$ million to $€ 0.22$ million per $\mathrm{GWh}$ in the dense scenario, while they range from $€ 0.04$ million to $€ 0.942$ million per GWh in the sparse scenario. This surplus stems especially from an increase in power plants, as a close inspection reveals that the 


\begin{tabular}{|c|c|c|c|c|c|c|c|c|}
\hline$N$ & Solver & $\begin{array}{l}\text { Total } \\
\text { time }(s)\end{array}$ & $\begin{array}{l}\text { Total cost } \\
(\mathrm{mn} \text { EUR/h) }\end{array}$ & $\begin{array}{l}\text { Variance of total } \\
\text { cost across runs }\end{array}$ & $\begin{array}{l}\text { Generation } \\
\text { cost (mn EUR/h) }\end{array}$ & $\begin{array}{l}\text { Transmission } \\
\text { cost (mn EUR/h) }\end{array}$ & $\begin{array}{c}\text { Relative cost } \\
(=\text { total cost } / \\
\left.\text { overall demand } \sum D_{i}\right)\end{array}$ & $\begin{array}{l}\text { Avg. plant } \\
\text { count }\end{array}$ \\
\hline \multirow{7}{*}{5} & Centralized heuristic & 0 & 0.064 & 0.003 & 0.052 & 0.012 & 0.077 & 1.0 \\
\hline & Distributed heuristic & 0 & 0.057 & 0.003 & 0.057 & 0.000 & 0.065 & 5.0 \\
\hline & Greedy heuristic & 0 & 0.057 & 0.003 & 0.054 & 0.003 & 0.064 & 3.0 \\
\hline & Neighborhood search (with centralized heuristic) & 1 & 0.055 & 0.003 & 0.051 & 0.004 & 0.063 & 2.7 \\
\hline & Neighborhood search (with distributed heuristic) & 1 & 0.053 & 0.003 & 0.052 & 0.002 & 0.058 & 3.3 \\
\hline & GRASP & 5 & 0.053 & 0.003 & 0.051 & 0.003 & 0.058 & 2.9 \\
\hline & Branch-and-reduce (GAMS/BARON) & 36 & 0.053 & 0.003 & 0.051 & 0.002 & 0.058 & 3.9 \\
\hline \multirow{7}{*}{10} & Centralized heuristic & 0 & 0.165 & 0.012 & 0.069 & 0.096 & 0.118 & 1.0 \\
\hline & Distributed heuristic & 1 & 0.080 & 0.001 & 0.080 & 0.000 & 0.061 & 10.0 \\
\hline & Greedy heuristic & 1 & 0.081 & 0.002 & 0.071 & 0.009 & 0.061 & 5.0 \\
\hline & Neighborhood search (with centralized heuristic) & 2 & 0.076 & 0.002 & 0.053 & 0.023 & 0.061 & 5.8 \\
\hline & Neighborhood search (with distributed heuristic) & 2 & 0.063 & 0.001 & 0.056 & 0.007 & 0.051 & 7.0 \\
\hline & GRASP & 8 & 0.061 & 0.001 & 0.041 & 0.020 & 0.049 & 5.8 \\
\hline & Branch-and-reduce (GAMS/BARON) & 48 & 0.057 & 0.001 & 0.040 & 0.017 & 0.045 & 7.3 \\
\hline \multirow{7}{*}{25} & Centralized heuristic & 2 & 0.857 & 0.063 & 0.202 & 0.655 & 0.244 & 1.0 \\
\hline & Distributed heuristic & 1 & 0.218 & 0.004 & 0.218 & 0.000 & 0.059 & 25.0 \\
\hline & Greedy heuristic & 1 & 0.230 & 0.005 & 0.187 & 0.043 & 0.063 & 13.0 \\
\hline & Neighborhood search (with centralized heuristic) & 4 & 0.289 & 0.026 & 0.137 & 0.152 & 0.073 & 14.0 \\
\hline & Neighborhood search (with distributed heuristic) & 5 & 0.181 & 0.004 & 0.155 & 0.026 & 0.048 & 17.6 \\
\hline & GRASP & 27 & 0.189 & 0.006 & 0.147 & 0.042 & 0.049 & 15.4 \\
\hline & Branch-and-reduce (GAMS/BARON) & 7260 & $0.157^{\dagger}$ & $0.004^{\dagger}$ & $0.124^{\dagger}$ & $0.033^{\dagger}$ & $0.041^{\dagger}$ & $16.6^{\dagger}$ \\
\hline \multirow{7}{*}{100} & Centralized heuristic & 16 & 7.526 & 4.475 & 0.549 & 6.977 & 0.491 & 1.0 \\
\hline & Distributed heuristic & 16 & 0.783 & 0.006 & 0.783 & 0.000 & 0.051 & 100.0 \\
\hline & Greedy heuristic & 19 & 1.065 & 0.023 & 0.616 & 0.449 & 0.070 & 50.0 \\
\hline & Neighborhood search (with centralized heuristic) & 22 & 1.807 & 0.682 & 0.626 & 1.181 & 0.118 & 60.8 \\
\hline & Neighborhood search (with distributed heuristic) & 55 & 0.684 & 0.006 & 0.604 & 0.079 & 0.045 & 70.6 \\
\hline & GRASP & 222 & 1.008 & 0.082 & 0.638 & 0.370 & 0.066 & 68.1 \\
\hline & Branch-and-reduce (GAMS/BARON) & 13,440 & $2.616^{\dagger}$ & $3.297^{\dagger}$ & $2.389^{\dagger}$ & $0.226^{\dagger}$ & $0.172^{\dagger}$ & $93.1^{\dagger}$ \\
\hline \multirow{7}{*}{200} & Centralized heuristic & 63 & 27.326 & 31.711 & 1.178 & 26.148 & 0.942 & 1.0 \\
\hline & Distributed heuristic & 61 & 1.537 & 0.028 & 1.537 & 0.000 & 0.053 & 200.0 \\
\hline & Greedy heuristic & 58 & 2.568 & 0.527 & 1.147 & 1.420 & 0.088 & 100.0 \\
\hline & Neighborhood search (with centralized heuristic) & 80 & 6.180 & 10.918 & 1.166 & 5.014 & 0.209 & 117.6 \\
\hline & Neighborhood search (with distributed heuristic) & 208 & 1.317 & 0.018 & 1.156 & 0.161 & 0.045 & 134.4 \\
\hline & GRASP & 847 & 2.356 & 0.066 & 1.211 & 1.145 & 0.081 & 140.4 \\
\hline & Branch-and-reduce (GAMS/BARON) & - & - & - & - & - & - & - \\
\hline
\end{tabular}

Table 6: These experiments compare the runtime and the characteristics of the solutions from the different optimization methods in the sparse scenario with $c_{\text {scale }}=125$. Results are averaged across 10 random instances. The variance of the retrieved objective (i. e. the total costs) across 10 runs is reported in order demonstrate the stability with which the different approaches generate solutions. The column with relative costs denotes the ratio of total cost to overall demand. It is thus independent of $N$ in order to compare the solutions across different instances of $N$. The legend additionally indicates experiments where solvers exceed our runtime limit of $2 \mathrm{~h}$ (i. e. 7,200s). The best solution approach for each $N$ is highlighted in bold. 
average number of plants rises. For instance, the branch-and-reduce baseline suggests an average of 2.3 plants in the dense scenario, while this number increases to 3.9 plants in the sparse scenario. At the same time, transmission costs decline from approx. $€ 16 \mathrm{k}$ to $€ 2 \mathrm{k}$ per hour. In summary, the sparse scenario demands solutions with a higher degree of distributed power generation.

\section{Case study: Optimization of the electricity infrastructure in Germany}

This section presents a real-world case study in which we apply our algorithms to the EGTP of Germany, with a peak demand of approximately 83.1 GW. For this purpose, we collected the locations of all $N=4,537$ municipalities. The corresponding demand is estimated by multiplying the relative population of each municipality by the previous peak demand per hour (see Figure 2). We again utilize the previous costs for power generation and transmission, as well as the same solver parameters.

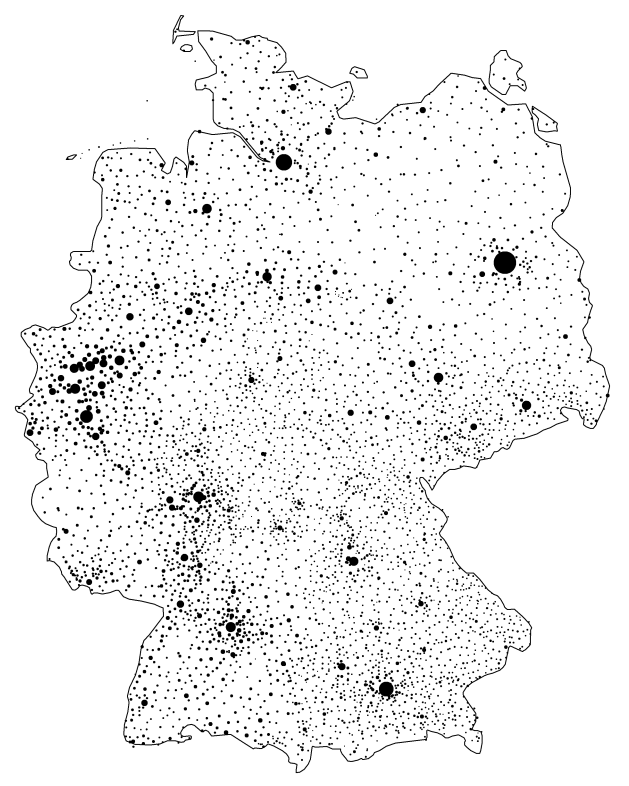

Figure 2: Points show locations of all 4,537 municipalities in Germany, with the point size indicating the electricity demand.

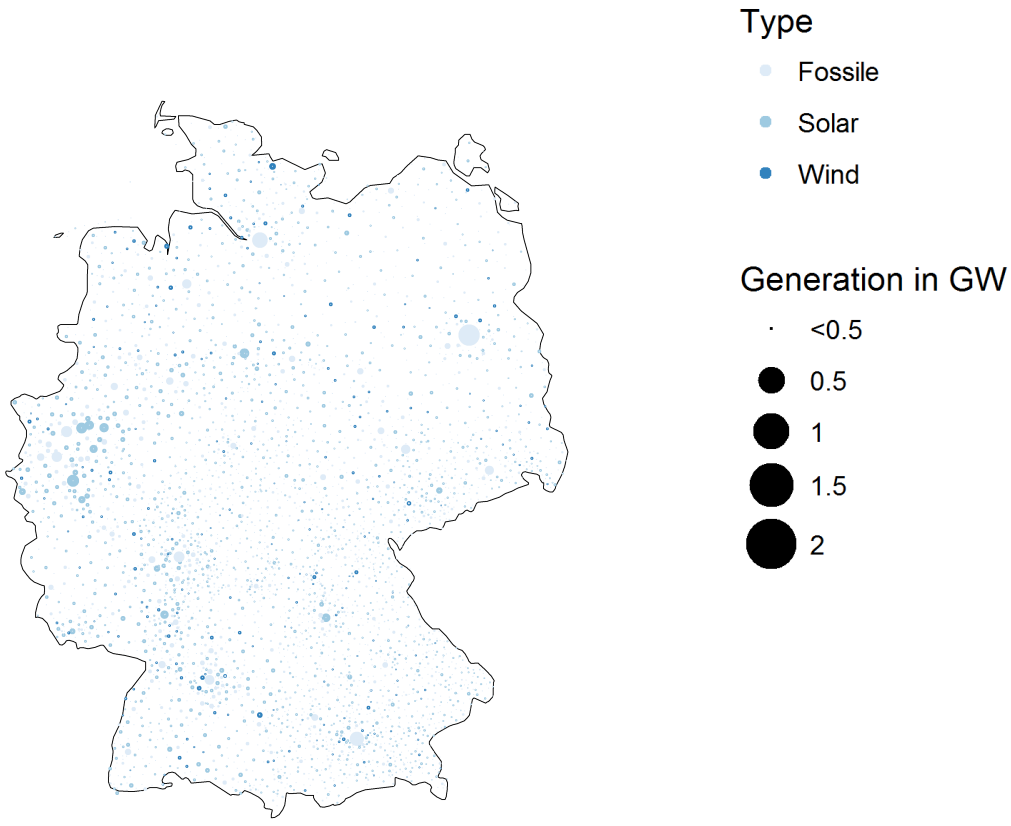

Figure 3: Solution computed by the reduced variable neighborhood search (with distributed heuristic) for the real-world case study.

Table 7 lists the results of our optimization methods. Detailed comparisons are provided in the online appendix. The reduced variable neighborhood search, together with the distributed construction heuristic, obtains the lowest total costs, amounting to $€ 5.1$ million per hour. The reduced variable neighborhood search solution is visualized in Figure 3 and it serves as the basis in 
all subsequent evaluations. The corresponding solution entails 2,385 power plants, which collectively yield an overall generation capacity of $83.1 \mathrm{GW}$. We notice several large-scale plants for brown coal and hard coal with capacities above $1 \mathrm{GW}$, as well as a large number of small and distributed power plants. These findings are comparable with the electricity infrastructure in Germany, where 1,618 power plants are currently in use.

\begin{tabular}{|c|c|c|c|c|c|c|c|}
\hline Solver & $\begin{array}{l}\text { Total } \\
\text { time }(\mathbf{h})\end{array}$ & $\begin{array}{l}\text { Total cost } \\
(\mathrm{mn} \text { EUR/h) }\end{array}$ & $\begin{array}{l}\text { Generation } \\
\text { cost }(\mathrm{mn} \text { EUR/h) }\end{array}$ & $\begin{array}{l}\text { Transmission } \\
\text { cost }(\mathrm{mn} \text { EUR/h) }\end{array}$ & $\begin{array}{l}\text { Number of } \\
\text { plants }\end{array}$ & $\begin{array}{l}\text { Avg. plant } \\
\text { capacity (GW) }\end{array}$ & $\begin{array}{l}\text { Power } \\
\text { transmitted }(\mathrm{GW})\end{array}$ \\
\hline Centralized heuristic & 13 & 33.667 & 6.452 & 27.215 & 1 & 83.100 & 79.540 \\
\hline Distributed heuristic & 15 & 8.062 & 8.062 & 0.000 & 4537 & 0.018 & 0.000 \\
\hline Greedy heuristic & 19 & 10.109 & 2.525 & 7.584 & 195 & 0.426 & 65.032 \\
\hline Neighborhood search (with centralized heuristic) & 110 & 13.006 & 3.237 & 9.769 & 1829 & 0.046 & 82.443 \\
\hline Neighborhood search (with distributed heuristic) & 114 & 5.096 & 4.886 & 0.210 & 2385 & 0.035 & 36.824 \\
\hline GRASP & 460 & 8.942 & 3.339 & 5.604 & 1857 & 0.044 & 79.733 \\
\hline
\end{tabular}

Table 7: Comparison of optimization methods for the real-world case study. The best solution approach is highlighted in bold.

We report further descriptive statistics pertaining to the abovementioned solution as follows (see Table 8). Evidently, at the nationwide level, electricity generation is dominated by solar power, followed by fossil sources. As expected, solar generation is driven by a large number of smallsized plants (i. e. 147 with an average capacity of $28.3 \mathrm{MW}$ ), whereas large-scale power plants (e.g. brown coal plants) are primarily placed in metropolitan areas with a high population density (e. g. North Rhine-Westphalia and cities that are distant from others such as Munich). Overall, the EGTP suggests a rather decentralized solution, especially when compared to the current electricity infrastructure in Germany. Notwithstanding, this suggestion is in line with current policy efforts that favor a decentralized distribution mix.

\begin{tabular}{lcccc}
\hline Generation type & Number of plants & Total capacity (GW) & Average capacity (MW) & SD \\
\hline Fossil, thereof: & 647 & 34.41 & 53.18 & 0.19 \\
Biogas & 573 & 14.93 & 26.05 & 0.04 \\
Brown coal & 50 & 9.56 & 191.16 & 0.27 \\
Hard coal & 10 & 3.06 & 305.99 & 0.43 \\
Gas & 14 & 6.86 & 490.08 & 0.88 \\
Solar & 1497 & 42.38 & 28.31 & 0.06 \\
Wind & 241 & 6.58 & 27.31 & 0.03 \\
\hline Total & 2385 & 83.37 & 34.96 & 0.11 \\
\hline
\end{tabular}

Table 8: Comparison of the distribution mix as computed in the EGTP, where SD refers to the standard deviation of the average capacity. 
We finally compare the solution from the EGTP against the spatial distribution of actual power plants in the wider area of Berlin (see Table 9). Specifically, we choose a rectangular area $50 \mathrm{~km}$ in length, which covers a population of approximately 4 million people with the largest cities being Berlin, Potsdam and Falkensee. Our optimization method has placed a total generation capacity of 4.1 GW in this area, which largely consists of fossil power $(3.9 \mathrm{GW})$ and, to a smaller extent, solar power $(0.2 \mathrm{GW})$. In comparison, the actual distribution of all power plants together in that area exhibits a smaller total generation capacity, which amounts only to $1.65 \mathrm{GW}$. It is stems largely from fossil sources (i.e. 1.55 GW), followed by solar (i. e. $0.05 \mathrm{GW}$ ) and wind (i. e. $0.06 \mathrm{GW}$ ). Hence, the solution proposed by EGTP can be considered rather self-sufficient as most generation facilities are located in close to the demand locations, while the actual solution is considerably more dependent on transmission. According to our optimization against the peak demand, the demand can reach up to $4.2 \mathrm{GW}$, in which case energy would need to be imported into that geographic region in the current grid layout, whereas our suggested layout could largely cover this demand without imports. We observe approximately the same distribution between fossil (approx. 94\%) and non-fossil generation capacities (approx. $6 \%$ for wind and solar). Furthermore, we point out that several of the locations in the actual grid layout (e.g. Berlin, Potsdam) overlap, while others are in close proximity (e.g. Kleinmachnow vs. Schönefeld). Similarly, we also see that the EGTP results in a large number of plants overall (11 vs. 7). Finally, we point out that some differences might be due to the fact that actual costs could potentially differ from those that we assumed in our model parameterization.

\begin{tabular}{|c|c|c|c|c|c|c|}
\hline \multirow{2}{*}{$\begin{array}{l}\text { Generation } \\
\text { type }\end{array}$} & \multicolumn{3}{|c|}{ Our EGTP solution } & \multicolumn{3}{|c|}{ Actual distribution } \\
\hline & Capacity (GW) & Share $(\%)$ & Locations & $\overline{\text { Capacity (GW) }}$ & Share $(\%)$ & Locations \\
\hline Fossil & 3.87 & 0.94 & $\begin{array}{l}\text { Berlin, Kleinmachnow, } \\
\text { Potsdam, Seddiner See, } \\
\text { Wustermark }\end{array}$ & 1.55 & 0.94 & $\begin{array}{l}\text { Berlin, Potsdam, } \\
\text { Schönefeld, Wilmersdorf }\end{array}$ \\
\hline Solar & 0.23 & 0.06 & $\begin{array}{l}\text { Dallgow-Döberitz, } \\
\text { Hoppegarten, Ludwigs- } \\
\text { felde, Mittenwalde, } \\
\text { Rangsdorf, Zeuthen }\end{array}$ & 0.05 & 0.03 & Berlin \\
\hline Wind & 0.00 & 0.00 & - & 0.06 & 0.04 & Berlin, Kleinmachnow \\
\hline Total & 4.10 & & & 1.65 & & \\
\hline
\end{tabular}

Table 9: Comparison of the distribution mix as computed in the EGTP (left) and in the actual region of Berlin, Germany. 


\section{Conclusion}

This paper proposes a novel electricity generation and transmission problem that aids practitioners and policy-makers in designing large-scale electricity systems. We consider the specific problem characteristics that arise in high-voltage transmission grids and that differ considerably from the extensive research on low-voltage distribution grids within municipalities, whereas highvoltage transmission grids have received only little attention. Our proposed problem models the combined costs for power generation and transmission infrastructure and specifically incorporates fixed upfront investments, as well as a variable, non-linear component. We show how to optimally solve this model using branch-and-reduce. However, the runtime of this approach ballons quickly for medium-sized instances with $N \geq 25$ locations. This renders it necessary that we develop heuristics in order to address problem instances that arise in practice with $N>1,000$. For this reason, we exploit the geometric structure and suggest different construction heuristics based on centralized and distributed power generation, as well as a locally-centralized variant with greedy properties. In addition, we devise an improvement heuristic that implements a reduced variable neighborhood search. Lastly, both are combined in a GRASP metaheuristic. All heuristics succeed in our goal of solving problem instances in a reasonable amount of time. This is finally demonstrated by our case study based on the nationwide electricity infrastructure in Germany.

The EGTP enables practitioners and policy-makers to infer decision support for designing electricity systems. It specifically helps in considering the intricate trade-off between extensive transmission infrastructure and highly distributed power generations (e. g. by renewable energy sources). The EGTP not only facilitates the design of brownfield settings as utilized in our computational experiments, but also allows for the study of greenfield setups by specifying the cost parameters accordingly, i.e. setting generation and transmission costs for existing facilities to zero or to the investments necessary for enlarging it.

The EGTP has manifold applications in practice, as it can also be utilized in use-cases where electricity infrastructure undergoes extensive modifications, e.g. due to policy goals or international treaties stipulating climate objectives. For instance, it can help in optimizing the electricity infrastructure in Germany after the nuclear power phase-out or the infrastructure in Japan in the wake of the Fukushima disaster. Altogether, this work provides valuable and powerful insights for decision-makers in the field of electricity systems.

Infrastructure planning in the electricity sector is subject to the inherent uncertainty of future developments, forecasted demands, and expected material cost. In practice, this is commonly 
addressed by scenario planning and, as shown earlier by comparing the variance across different numerical experiments for the same grid size, our EGTP model can be naturally leveraged in such cases. Its formalization, as well as the corresponding optimization routines, are intentionally designed so that they foster extensibility and, analogous to Snyder (2006), could be used when extending this work into a stochastic variant of the EGTP.

In future work, the EGTP might be extended by additional constraints, such as a certain energy mix or production quota for renewable energy sources in order to satisfy regulatory obligations. It is noteworthy that the EGTP can easily adapt to regional differences in costs; for instance, it can take into account the fact that certain regions are more suited to solar or wind power than others. Finally, it is straightforward to adapt the problem to variable renewable energy sources (VRES) or peak load curves by making the supply and demand variables time-dependent, simply by adding an additional index. Moreover, practitioners might desire to repeat the analysis for different hours of the day (or a year) in order to identify a solution that can live up to the requirements of a peak load scenario.

\section{References}

Adenso-Díaz, B., \& Rodríguez, F. (1997). A simple search heuristic for the MCLP: Application to the location of ambulance bases in a rural region. Omega, 25, 181-187.

Albareda-Sambola, M., Fernández, E., \& Nickel, S. (2012). Multiperiod location-routing with decoupled time scales. European Journal of Operational Research, 217, 248-258.

Antunes, C., Martins, A., \& Brito, I. S. (2004). A multiple objective mixed integer linear programming model for power generation expansion planning. Energy, 29, 613-627.

Antunes, C. H., \& Gomes, Á. (2009). Operational research models and methods in the energy sector. European Journal of Operational Research, 197, 997-998.

Baringo, L., \& Conejo, A. J. (2012). Wind power investment: A benders decomposition approach. IEEE Transactions on Power Systems, 27, 433-441.

Baumgartner, K., Fuetterer, A., \& Thonemann, U. W. (2012). Supply chain design considering economies of scale and transport frequencies. European Journal of Operational Research, 218, 789-800.

Bemis, G. R., \& DoAngelis, M. (1990). Levelized cost of electricity generation technologies. Contemporary Economic Policy, 8, 200-214.

Billionnet, A., Costa, M.-C., \& Poirion, P.-L. (2016). Robust optimal sizing of a hybrid energy stand-alone system. European Journal of Operational Research, 254, 565-575. 
Black and Veatch (2011). Cost and performance data for power generation technologies. Cost report for the national renewable energy laboratory, https://www.bv.com/docs/reports-studies/nrel-cost-report.pdf.

Boomsma, T. K., Meade, N., \& Fleten, S.-E. (2012). Renewable energy investments under different support schemes: A real options approach. European Journal of Operational Research, 220, 225-237.

Bundesnetzagentur (2016). Monitoring report 2016. Online: https://www.bundesnetzagentur.de/ SharedDocs/Downloads/DE/Sachgebiete/Energie/Unternehmen_Institutionen/Datenaustausch UndMonitoring/Monitoring/Monitoring2016/Monitoring Summary2016.pdf (visited on 04/14/2017), .

Capros, P., Papathanassiou, S., \& Samouilidis, J. E. (1988). Multicriteria analysis of energy supply decisions in an uncertain future. Omega, 16, 107-115.

Chen, S.-G. (2013). Bayesian approach for optimal pv system sizing under climate change. Omega, 41, $176-185$.

Christensen, L. R., \& Greene, W. H. (1976). Economies of scale in U.S. electric power generation. Journal of Political Economy, 84, 655-676.

Conejo, A. J., Siddiqui, A. S., Kazempour, S. J., \& Baringo Morales, L. (2016). Investment in Electricity Generation and Transmission: Decision Making under Uncertainty. Cham, Switzerland: Springer.

Darvish, M., Larrain, H., \& Coelho, L. C. (2016). A dynamic multi-plant lot-sizing and distribution problem. International Journal of Production Research, 54, 6707-6717.

Delucchi, M. A., \& Jacobson, M. Z. (2011). Providing all global energy with wind, water, and solar power, part II: Reliability, system and transmission costs, and policies. Energy Policy, 39, 1170-1190.

Dornburg, V., \& Faaij, A. P. (2001). Efficiency and economy of wood-fired biomass energy systems in relation to scale regarding heat and power generation using combustion and gasification technologies. Biomass and Bioenergy, 21, 91-108.

Duhamel, C., Lacomme, P., Prins, C., \& Prodhon, C. (2010). A GRASPxELS approach for the capacitated location-routing problem. Metaheuristics for Logistics and Vehicle Routing, 37, 1912-1923.

Ernstsen, R. R., \& Boomsma, T. K. (2018). Valuation of power plants. European Journal of Operational Research, 266, 1153-1174.

Feldman, E., Lehrer, F. A., \& Ray, T. L. (1966). Warehouse location under continuous economies of scale. Management Science, 12, 670-684.

Feo, T. A., \& Resende, M. G. C. (1995). Greedy randomized adaptive search procedures. Journal of Global Optimization, 6, 109-133.

Fleten, S., Maribu, K., \& Wangensteen, I. (2007). Optimal investment strategies in decentralized renewable power generation under uncertainty. Energy, 32, 803-815.

Ghiani, G., Guerriero, F., \& Musmanno, R. (2002). The capacitated plant location problem with multiple facilities in the same site. Computers \& Operations Research, 29, 1903-1912. 
Golari, M., Fan, N., \& Jin, T. (2017). Multistage stochastic optimization for production-inventory planning with intermittent renewable energy. Production and Operations Management, 26, 409-425.

Grimm, V., Martin, A., Schmidt, M., Weibelzahl, M., \& Zöttl, G. (2016). Transmission and generation investment in electricity markets: The effects of market splitting and network fee regimes. European Journal of Operational Research, 254, 493-509.

Guo, Z., Ma, L., Liu, P., Jones, I., \& Li, Z. (2016). A multi-regional modelling and optimization approach to China's power generation and transmission planning. Energy, 116, 1348-1359.

Gupta, A., Saini, R. P., \& Sharma, M. P. (2011). Modelling of hybrid energy system-part i: Problem formulation and model development. Renewable Energy, 36, 459-465.

Hansen, P., Mladenović, N., Brimberg, J., \& Pérez, J. A. M. (2010). Variable neighborhood search. In M. Gendreau, \& J.-Y. Potvin (Eds.), Handbook of Metaheuristics (pp. 61-86). Boston, MA: Springer volume 146 of International Series in Operations Research 83 Management Science.

Hobbs, B. F. (1995). Optimization methods for electric utility resource planning. European Journal of Operational Research, 83, 1-20.

Hobbs, B. F., \& Ji, Y. (1999). Stochastic programming-based bounding of expected production costs for multiarea electric power system. Operations Research, 47, 836-848.

Huppmann, D., \& Egerer, J. (2015). National-strategic investment in european power transmission capacity. European Journal of Operational Research, 247, 191-203.

Jarboui, B., Derbel, H., Hanafi, S., \& Mladenović, N. (2013). Variable neighborhood search for location routing. Computers \& Operations Research, 40, 47-57.

Kocuk, B., Jeon, H., Dey, S. S., Linderoth, J., Luedtke, J., \& Sun, X. A. (2016). A cycle-based formulation and valid inequalities for DC power transmission problems with switching. Operations Research, 64, 922-938.

Krarup, J., \& Pruzan, P. M. (1983). The simple plant location problem: Survey and synthesis. European Journal of Operational Research, 12, 36-81.

Kumar, M., Kumar, A., \& Sandhu, K. S. (2016). WT-based distributed generation location minimizing transmission loss using mixed integer nonlinear programming in deregulated electricity market. In S. C. Satapathy, Y. C. Bhatt, A. Joshi, \& D. K. Mishra (Eds.), Proceedings of the International Congress on Information and Communication Technology (pp. 443-458). Singapore: Springer volume 438 of Advances in Intelligent Systems and Computing.

Lohmann, T., \& Rebennack, S. (2016). Tailored benders decomposition for a long-term power expansion model with short-term demand response. Management Science, 63, 1657-2048.

Manne, A. S. (1964). Plant location under economies-of-scale: Decentralization and computation. Management Science, 11, 213-235. 
Marinakis, V., Doukas, H., Xidonas, P., \& Zopounidis, C. (2017). Multicriteria decision support in local energy planning: An evaluation of alternative scenarios for the sustainable energy action plan. Omega, 69, 1-16.

Meijboom, B., \& Obel, B. (2007). Tactical coordination in a multi-location and multi-stage operations structure: A model and a pharmaceutical company case. Omega, 35, 258-273.

Meneses de Quevedo, P., \& Contreras, J. (2016). Optimal placement of energy storage and wind power under uncertainty. Energies, 9, 528 .

Mínguez, R., \& García-Bertrand, R. (2016). Robust transmission network expansion planning in energy systems: Improving computational performance. European Journal of Operational Research, 248, 21-32.

Mustakerov, I., \& Borissova, D. (2010). Wind turbines type and number choice using combinatorial optimization. Renewable Energy, 35, 1887-1894.

Nara, K., Hayashi, Y., Ikeda, K., \& Ashizawa, T. (2001). Application of tabu search to optimal placement of distributed generators. In Winter Meeting of the IEEE Power Engineering Society 2001 (pp. 918-923).

Nickel, S., \& da Gama, F. S. (2015). Multi-period facility location. In G. Laporte, S. Nickel, \& F. Saldanha da Gama (Eds.), Location Science (pp. 289-310). Cham: Springer International Publishing.

Parpas, P., \& Webster, M. (2014). A stochastic multiscale model for electricity generation capacity expansion. European Journal of Operational Research, 232, 359-374.

Paul, N. R., Lunday, B. J., \& Nurre, S. G. (2017). A multiobjective, maximal conditional covering location problem applied to the relocation of hierarchical emergency response facilities. Omega, 66, $147-158$.

Pineda, S., Boomsma, T. K., \& Wogrin, S. (2018). Renewable generation expansion under different support schemes: A stochastic equilibrium approach. European Journal of Operational Research, 266, 1086-1099.

Pozo, D., Sauma, E. E., \& Contreras, J. (2013). A three-level static MILP model for generation and transmission expansion planning. IEEE Transactions on Power Systems, 28, 202-210.

Prodhon, C., \& Prins, C. (2014). A survey of recent research on location-routing problems. European Journal of Operational Research, 238, 1-17.

Qi, W., Liang, Y., \& Shen, Z.-J. M. (2015). Joint planning of energy storage and transmission for wind energy generation. Operations Research, 63, 1280-1293.

Resende, M. G., \& Ribeiro, C. C. (2010). Greedy randomized adaptive search procedures: Advances, hybridizations, and applications. In M. Gendreau, \& J.-Y. Potvin (Eds.), Handbook of Metaheuristics (pp. 283-319). Boston, MA: Springer US volume 146 of International Series in Operations Research 83 Management Science.

RMI (2011). Present value cost of the U.S. electricity system. Online: http://www.rmi.org/RFGraphpresent_value_cost_US_electricity (visited on 04/14/2017), .

Rossi, A., Aubry, A., \& Jacomino, M. (2012). Connectivity-and-hop-constrained design of electricity distri- 
bution networks. European Journal of Operational Research, 218, 48-57.

Sabet, E., Yazdani, B., Kian, R., \& Galanakis, K. (2019). A strategic and global manufacturing capacity management optimisation model: A scenario-based multi-stage stochastic programming approach. Omega,

Samouilidis, J.-E., Psarras, J., \& Papaconstantinou, D. V. (1984). Electricity planning vs energy planning: A modelling approach. Omega, 12, 341-346.

Schiffer, M., \& Walther, G. (2018). Strategic planning of electric logistics fleet networks: A robust locationrouting approach. Omega, 80, 31-42.

Seddighi, A. H., \& Ahmadi-Javid, A. (2015). Integrated multiperiod power generation and transmission expansion planning with sustainability aspects in a stochastic environment. Energy, 86, 9-18.

Sherali, H. D., \& Staschus, K. (1990). A two-phase decomposition approach for electric utility capacity expansion planning including nondispatchable technologies. Operations Research, 38, 773-791.

Singh, J. (1990). Production planning for thermal electric power industry of the eastern india (mixed-integer programming approach). Indian Economic Review, 25, 105-136.

Snyder, L. V. (2006). Facility location under uncertainty: A review. IIE Transactions, 38, 547-564.

Sovacool, B. K., Nugent, D., \& Gilbert, A. (2014). Construction cost overruns and electricity infrastructure: An unavoidable risk? The Electricity Journal, 27, 112-120.

Sridharan, R. (1995). The capacitated plant location problem. European Journal of Operational Research, $87,203-213$.

Stolzenberger, C. (2015). Levelised cost of electricity. Online: https://www.vgb.org/en/lcoe2015.html?dfid=74042 (visited on 10/18/2016), .

Sun, H., Yang, J., \& Yang, C. (2018). A robust optimization approach to multi-interval location-inventory and recharging planning for electric vehicles. Omega, .

Tawarmalani, M., \& Sahinidis, N. V. (2005). A polyhedral branch-and-cut approach to global optimization. Mathematical Programming, 103, 225-249.

Thrampoulidis, C., Bose, S., \& Hassibi, B. (2015). Optimal placement of distributed energy storage in power networks. IEEE Transactions on Automatic Control, 61, 416-429.

van der Weijde, A. H., \& Hobbs, B. F. (2012). The economics of planning electricity transmission to accommodate renewables: Using two-stage optimisation to evaluate flexibility and the cost of disregarding uncertainty. Energy Economics, 34, 2089-2101.

Vasin, A., \& Dolmatova, M. (2016). Optimization of transmission capacities for multinodal markets. Procedia Computer Science, 91, 238-244.

Villumsen, J. C., \& Philpott, A. B. (2012). Investment in electricity networks with transmission switching. European Journal of Operational Research, 222, 377-385. 
Walla, C., \& Schneeberger, W. (2008). The optimal size for biogas plants. Biomass and Bioenergy, 32, $551-557$.

Wang, T., \& Deng, S. (2018). Multi-period energy procurement policies for smart-grid communities with deferrable demand and supplementary uncertain power supplies. Omega, forthcoming.

Zhan, Y., Zheng, Q. P., Wang, J., \& Pinson, P. (2017). Generation expansion planning with large amounts of wind power via decision-dependent stochastic programming. IEEE Transactions on Power Systems, 32, 3015-3026.

Zugno, M., \& Conejo, A. J. (2015). A robust optimization approach to energy and reserve dispatch in electricity markets. European Journal of Operational Research, 247, 659-671. 